

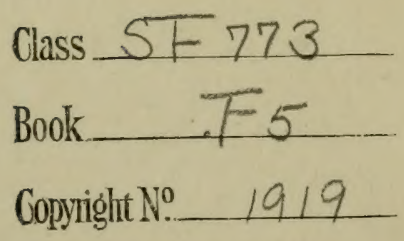

COFYRIGHT DEPOSIT: 








\title{
THE
}

\section{Examination of the Urine}

\author{
OF THE
}

\section{HORSE AND MAN}

BY

\section{PIERRE A. FISH, D.Sc., D.V.M.}

\section{PROFESSOR OF VETERINARY PHYSIOLOGY}

NEW YORK STATE VETERINARY COLLEGE

CORNELL UNIVERSITY

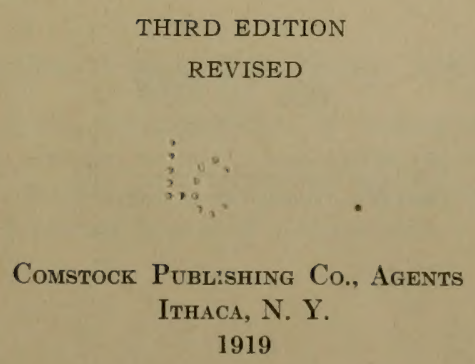




\section{SF773 \\ . 55 \\ 1919}

\section{Copyright 1919}

by

PIERRE A. FISH

DEC 101919

PRESS OF W. F. HUMPHREY, GENEYYAS R. Y.

(C)Cl:A5 36984

no 1 


\section{PREFACE.}

This manual represents the published form of mimeographed sheets, which the writer has used, for some years past, in giving instruction to his veterinary students. Data relating to the urine of the horse is not abundant and, unfortunately, some of the tests which may be standard for human urine are not reliable for that of the horse and, therefore, require modification. In order to emphasize this fact, the writer has employed the comparative method and asks the students to examine their own urine along with that of the horse and note carefully the differences in the results.

The importance of urine examination for diagnosis and prognosis in human medicine is too well known to require emphasis here; but, notwithstanding certain difficulties in the way of its application in veterinary medicine, the writer believes that there is no important reason why just as much valuable information may not be derived, in certain cases, from the urine of the horse by an up-to-date veterinarian, as the physician obtains from the urine of his patients.

Simplification of 'methods, without too great a sacrifice in accuracy, is essential for satisfactory examination,- -especially by veterinarians. The writer realizes that only a short step has been taken in this direction, but hopes that time will lighten the difficulties.

OCTOBER, 1906.

P. A. F.

\section{PREFACE TO THE SECOND EDITION}

In the present edition some changes have been made in the text and new material added with the hope of bringing the manual more completely up to date. There is still much to be done in investigating and simplifying the tests applicable to the urine of the domesticated animals and it is to be hoped that more research may be developed along this line.

NONeMber, 1911.

P. A. F.

\section{PREFACE FOR THE THIRD EDITION}

Experience has shown the desirability of making certain changes and some additions to the previous edition in order to bring this work up to date. Acknowledgement is gladly made to Dr. C. E. Hayden and former students whose suggestions and interest have been of much value

MARCH, 1919.

$$
\text { P. A. F. }
$$


CHAPTER I.

The Secretion of Urine. . . . . . . . . . . . . .

CHAPTER II.

Quantity, Color, Transparency, Consistency, Reaction,

Degree of Acidity, Specific Gravity..............pp. 12-19

CHAPTER III.

Qualitative tests: Inorganic Constituents, Water,

Chlorides, Sulphates, Phosphates, Carbonates....pp. 19-24

CHAPTER IV.

Organic Constituents: Urea, Uric Acid, Hippuric

Acid, Creatinin, Mucus, Indican, Oxalic Acid,

Acetone, Diacetic Acid, Urobilin, Leucin, Tyrosin,

Phenol ........................... pp. 24-33

CHAPTER V.

Abnormal Substances in the Urine: Albumin, Sugar...pp. 33-41 CHAPTER VI.

Bile, Blood, Melanine......................... 41-45

CHAPTER VII.

Quantitative Analysis, Centrifugal Method: Phosphates, Chlorides, Sulphates, Uric Acid, Uricometer, Urea, Albumin, Saccharometer, Sugar, Ehrlich's Diazo-reaction.................... pp. 45-56

CHAPTER VIII.

Volumetric Methods: Chlorides, Phosphoric Acid, Sulphuric Acid, Relation of Constituents in Normal Urine pp. 56-59 CHAPTER IX.

Chemical Examination of Urinary Deposits, Acid Urate of Soda, Uric Acid, Cystine, Pus, Blood, A1kaline Urates, Triple Phosphates, Calcium Oxalate, Calcium Carbonate, Pus, Blood.............. pp. 59-62

CHAPTER X.

Microscopical Examination of Urine, Unorganized Sediments: Uric Acid, Acid Urate of Soda, Oxalate of Lime, Hippuric Acid, Calcium Sulphate, Calcium Phosphate, Triple Phosphate, Urate of Ammonium, Cystin, Leucin, Tyrosin. Organized Sediments: Epithelial cells; Glycogen cells, Amyloid bodies, Hyaline, Granular, Epithelial, Fat, Hemorrhagic and Waxy casts; Cylindroids; Blood and Pus corpuscles; Spermatozoa; Mucus; Bacteria.........pp. 62-73 Form for Urine Examinations, Procedure in Examining

a sample of urine. . . . . . . . . . . . . . . . pp. 74-77 Appendix, Formulae for Reagents.............pp. $77-80$ 


\section{URINE ANALYSIS.}

APPARATUS FOR THE LOCKER

1 dozen test tubes, 6 inch

1 dozen test tubes, 5 inch

1 Minim pipette

1 Beaker, 10 oz.

1 Graduate, $30 \mathrm{cc}$.

1 Graduate, $250 \mathrm{cc}$.

1 Flask

1 Funnel, $1 \mathrm{I} / 2$ inch

1 Funnel, 3 inch

1 Watch glass

1 Evaporating dish, $8 \mathrm{oz}$.

1 Urinsmeter

1 Glass rod

1 Thermometer

1 Crucible, 8 cc.
1 Piece wire gauze

1 Piece absorbent cotton

1 Box matches

1 Test tube brush

1 Test tube rack

1 Test tube holder, wire

1 Tripod

1 Piece muslin

1 Pack filter papers, 3 inch

1 Pack filter papers, 6 inch

1 Sponge

1 Clay triangle

2 Tin cans

1 Copper water bath

1 Towel

Special apparatus, not found in the locker, may be obtained, when needed, by handing an order for it to one of the assistants. 



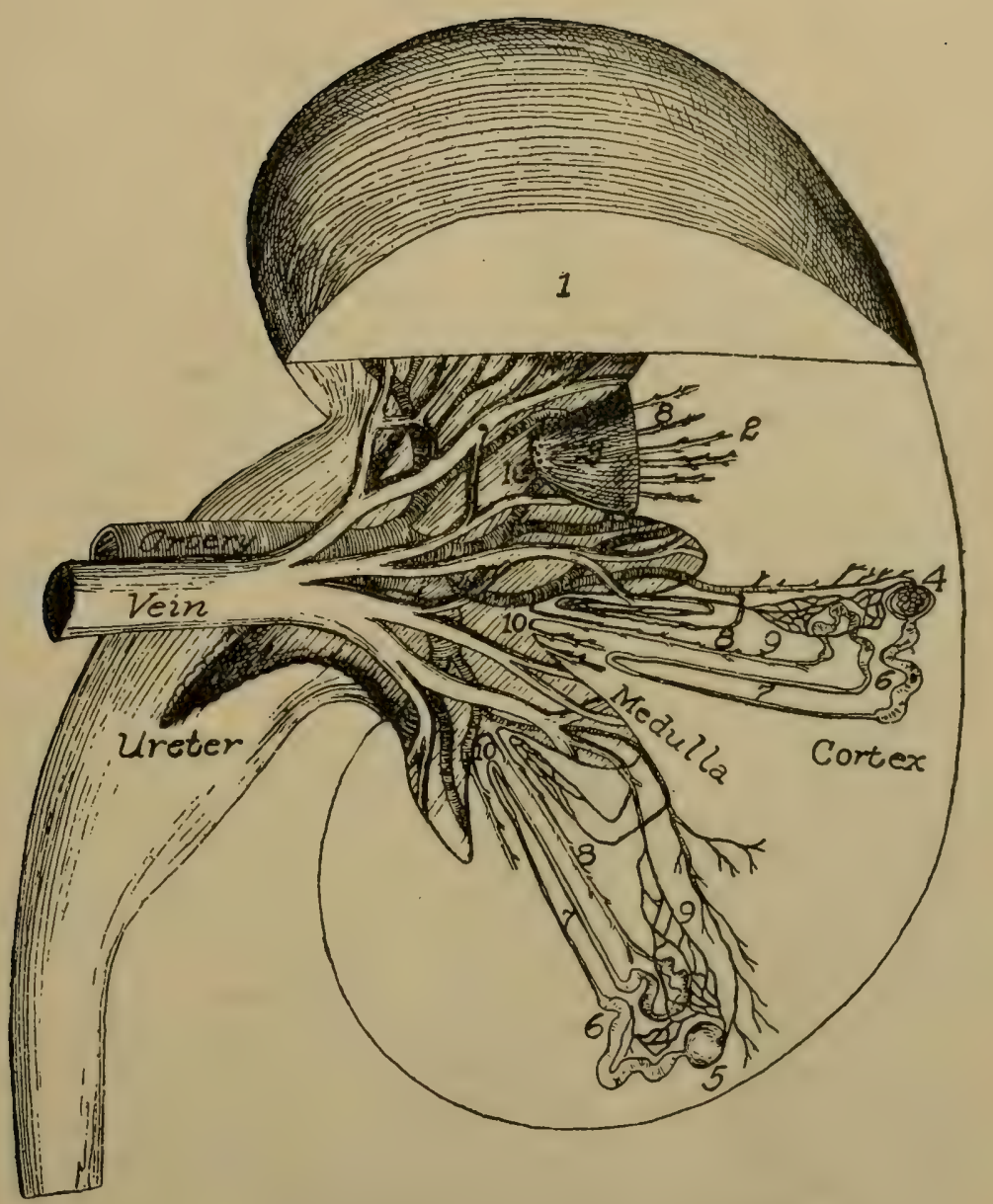

Plate 1. Schematic Section of the Kidney

1. Cut portion of the Kidney. 2. Pyramid. 3. Papilla of the Pyramid. 4. Glomerulus, enclosed in Bowman's Capsule. 5. Bowman's Capsule. 6. Convoluted portion of Renal Tubule. 7. Loop of Henle. 8. Collecting Tubule. 9. Capillary net work,- - second set; the first set is the Glomerulus. 10. Openings of Collecting Tubules on Papillae, from which the Urine drops into the Pelvis of the Kidney. 


\section{THE SECRETION OF URINE.}

I.

The blood supply to the kidneys is an important consideration in the secretion of urine. At the outset, one is impressed by the fact that a relatively large artery supplies a relatively small gland. The blood pressure of the renal artery is nearly, if not quite, as great as that of the abdominal aorta, which means that about as much pressure is required to force the blood through the kidneys as is required to send the blood through the pedal extremities. The renal vein is also a relatively large vessel as compared with the size of the kidney or with the efferent vessels of other glands. The pressure within the renal vein is very low, practically as low as that in the posterior vena cava with which it connects.

The artery, after entering the kidney, breaks up into branches which pass between the pyramids. At the junction of the cortex and medulla these branches form arches in the substance of the kidneys and from these arches branches run outward into the cortex to supply the glomeruli; other branches pass inward to supply the pyramids. Each glomerulus has its afferent and efferent vessel and of these the efferent is smaller.

On issuing from the capillaries of the glomerulus, the efferent vessel soon breaks up into a second set of capillaries which supplies the uriniferous tubules; so that in this arrangement the blood goes first to the glomeruli and later supplies the tubules. The blood from the second set of capillaries is finally gathered up by'small vessels which unite to form ultimately the renal vein. The glomeruli and the uriniferous tubules are the portions of the kidney actively concerned in the production of its secretionthe urine.

The following points are therefore worthy of special notice; in the malpighian body the arterial blood in the glomerular capillaries is separated from the inside of the capsule of Bowman by a thin layer of flattened epithelial cells only; two sets of capillaries exist, one set forming the glomeruli and the other supplying the uriniferous tubules, the blood supplying the tubules 

must have first passed through the glomeruli, and is therefore more concentrated; in certain portions of the tubules short cylindrical epithelial cells are found which are comparable to true secreting cells found in other glands; and finally, the smaller size of the efferent as compared with the afferent vessel fulfills a condition which retards the flow of blood through the glomerulus.

The conditions are favorable for a high and variable pressure in the glomerulus and for a lower and more constant pressure in the second set of capillaries around the uriniferous tubules: On account of the resistance offered by a double system of capillaries the blood pressure in the kidneys is kept relatively high.

The changes in blood pressure may be observed upon the

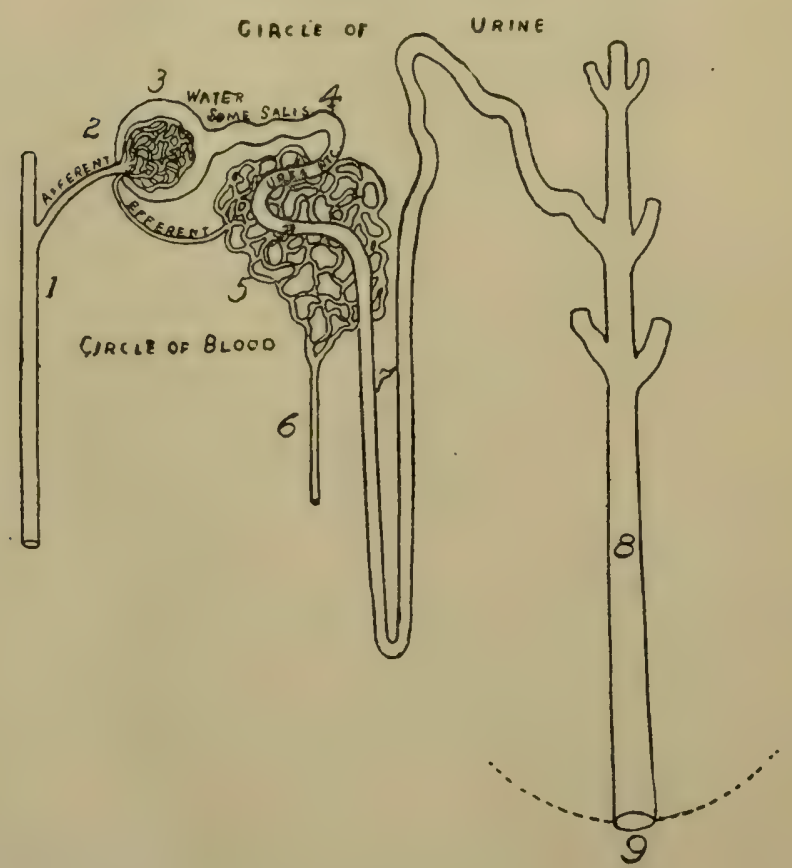

FIG. 1

1. Artery. 2. Glomerulus. 3. Capsule of Bowman. 4. Convoluted portion of Tubule. 5. Capillary net work. 7. Loop of Henle. 8. Collecting Tubule. 9. Opening of Tubule on Papilla. 

kidney itself by means of an apparatus known as the oncometer. With each rise in the blood pressure the kidney swells, with each fall in pressure it contracts, and a tracing can be obtained very similar to an ordinary blood pressure tracing. The glomerulus suspended in its capsule is also influenced by these changes. When the capillaries are engorged with blood the glomerulus fills the capsule, when collapsed there is an evident space between the membranes of the glomerulus and the capsule.

In 1842 Bowman advanced a theory relating to the production of urine which, while recognizing to some extent certain physical factors, also brought out the view that the urine is a secretion.

In 1844 Ludwig advanced the theory, sometimes referred to as the mechanical theory, in which only physical processes were involved, $i . e$., filtration, diffusion and osmosis. It is true that in the capillaries of the glomerulus there is a high resistance because of the smaller size of the efferent vessel, there is, therefore, a higher degree of pressure in the glomerulus than in the capsule in which it is suspended. This inequality of pressure is favorable to filtration. As the result of any filtration from the glomerulus some of the water of the blood with a certain proportion of dissolved substances would pass into the beginning of the uriniferous tubule. The effect of such a filtration would render the blood remaining in the glomerulus and second set of capillaries more concentrated, and in the second set of capillaries in connection with the uriniferous tubules the essential elements of an osmometer would be obtained,-an animal membrane formed by the delicate wall of the capillary and the wall of the tubule, upon one side of which there is a dense fluid, the blood, and upon the other a weak saline solution, conditions which are favorable to the processes of osmosis and diffusion.

In this theory, as a result of the interchange of these fluids, some of the water passes from the tubule to the blood, making it less concentrated, and the products of retrogressive metamorphosis,- - urea and other organic substances,- - pass from the blood to the tubule, forming urine. An objection in connection with the diffusion of urea has been raised in that it is a well-known fact that the urine contains a much greater amount of urea than does the blood and that it would be contrary to the laws 

of osmosis and diffusion for a fluid weak in urea (the blood) to pass through a membrane a substance which has accumulated in greater amount in a fluid (the urine) on the other side of that

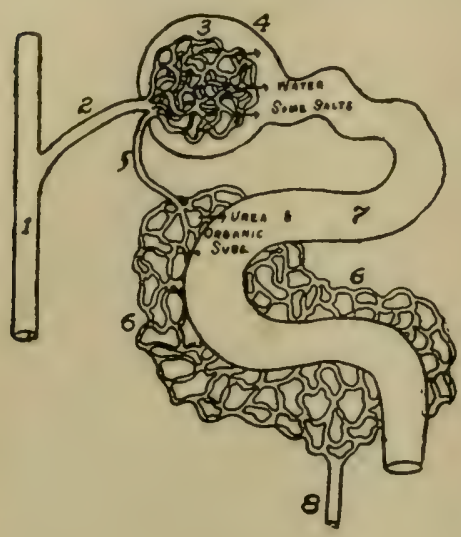

FIG. 2

1. Artery. 2. Afferent Vessel. 3. Glomerulus. 4. Capsule of Bowman. 5. Efferent Vessel. 6. Capillary network. 7. Uriniferous Tubule. 8. Vein. membrane. It should also be remembered that the blood is constantly moving and presumably the fluid in the tubules is doing the same. In other words, it would seem that if the conditions indicate an osmometer, it varies from experimental ones in that the fluids on either side of the membrane are moving in a definite direction. The proteid constituents do not normally leave the blood on account of their well-known indisposition to osmosis.

One important fact, however, remains unaccounted for in

Ludwig's mechanical theory, and that is, if the uriniferous tubules are stripped of their epithelial cells, as they often are in disease, urea and some other nitrogenous products are no longer, or only imperfectly eliminated, and become stored up in the blood and produce the condition known as uremia, although the conditions of an osmometer remain. It must, therefore, be admitter that there is some direct or elaborating action on the part of the epithelium as originally suggested by Bowman, although under normal conditions, transudation, diffusion and osmotic processes may occur coincidently.

The theoretical conclusions of Bowman have been confirmed and extended by the practical researches of Heidenhain, who injected indigo-carmine into the blood of animals and found that it was promptly removed by the kidneys. These organs were removed at suitable intervals after the injection and carefully examined under the microscope. In no instance did he find any of the indigo in the capsules of Bowman; but it was found abundantly in the cells lining certain portions of the tubules, and in the lumen of the tubules near these cells. Similar experiments 

with the urate of sodium showed that it likewise was secreted at the same place and in the same manner.

Further investigations have tended to diminish the importance of the "mechanical" factor and to develop a selective or secretory function for the cells, even including those of the glomeruli, by virtue of which the cells will permit certain substances to pass through and prevent others, among the latter, albumin. The view is advanced that the passage of the fluid through the glomerulus is not a mere transudation, but a matter of selection also.

The selective action of the renal cells is both qualitative and quantitative. As an example of the former, it is shown by experiment that if some egg albumin be injected into the blood, it is promptly eliminated by the kidneys. Egg albumin is not very markedly different from the serum albumin of the blood; both are indiffusible, but the renal cells recognize the former as a foreign substance and immediately separate it from the blood. The sugar, in normal quantity in the blood, although a diffusible substance, is not selected. Urea, which is also diffusible, but existing in the blood in much smaller amount than sugar, is selected and appears in the urine. Why this should be so it is difficult to explain, although it is a well-known fact that the sugar serves as a food for the tissues and is needed by the system, while urea is a waste product and would be detrimental to the system if not eliminated.

That the cells exert a quantitative selection is shown by the fact that when sugar is present in the blood in excessive amounts, 3 parts per 1000 or over, the excess is promptly eliminated.

Diet influences the reaction of the urine. A vegetable diet favors an alkaline reaction; a diet of flesh favors an acid reaction. The urine of the herbivora is therefore alkaline, while in the carnivora and omnivora the urine is mainly acid, although influenced to some extent by the kind of food eaten. The formation of an acid fluid from alkaline material,- - blood and lymph,is at first sight puzzling. The well-known fact that the gastric secretion of all mammals is acid is a case in point. Furthermore, experimental evidence shows that if an alkaline solution of sodium bicarbonate be placed upon one side of the membrane in an osmometer and a solution of neutral sodium phosphate be 

placed upon the other side and a weak electric current be sent through the solutions, the fluid in contact with the positive pole will, in a short time, become acid from the formation of acid sodium phosphate, while the fluid in contact with the negative pole is increased in alkalinity $\mathrm{Na} \mathrm{HCO}_{3}+\mathrm{Na}_{2} \mathrm{HPO}_{4}=\mathrm{Na}_{2} \mathrm{CO}_{3}+\mathrm{Na}$ $\mathrm{H}_{2} \mathrm{PO}_{4}$.

While it may be true to a considerable extent that the kidney merely removes certain constituents from the blood and transfers them to the urine, it has been shown that the activity of the renal cells is required in the production of hippuric acid, -a new product, as hippuric acid is not present in the blood.

In general, the theory explaining the secretion of urine, according to observed facts, is 'one which, while recognizing the process as partly physical, also requires some process of activity or elaboration on the part of the kidney itself.

\section{II.}

The urine of all mammals may be regarded, for the most part, as a solution of constituents derived from the metabolism of the tissues of the body. Some of these constituents, especially the inorganic, may appear in the urine in the same form as they are taken into the body in the food, e. g., sodium chloride; others, especially the organic, represent decomposition products derived from the food or tissues, e. g., urea, creatinin, etc. The composition of the urine may, therefore, to some extent be regarded as an index of tissue activity, and the examination of this secretion is of considerable importance in clinical diagnosis and prognosis.

That there is a relationship between the diet and the renal excretion is shown by the examination of the urine of the three great classes of animals grouped according to the food they eat; herbivora, omnivora and carnivora. Perhaps the first and most striking characteristic of the urine of each group is its chemical reaction. In vegetable feeders it is normally alkaline; in flesh eaters it is markedly acid, and in the omnivora it may be acid or alkaline according to the preponderance of the fleshy or vegetable material in the food.

The relative proportion of phosphoric and carbonic acids 

in the blood depends very much upon the composition of the diet. The chief mineral constituents of animal food are phosphates; those of vegetable foods carbonates. The blood of herbivorous animals is therefore rich in the latter, that of the carnivora in the former, and the acids formed in the tissues will form bicarbonates in the blood of the herbivora, and acid phosphates in that of carnivora. Acid phosphates hold earthy phosphates in solution, and bicarbonates dissolve earthy carbonates. Hence the urine of carnivorous animals is rich in phosphates of lime and magnesia, that of the herbivora in calcium and magnesium carbonate.

In the practical treatment of this subject it is convenient to regard the horse as the type for the herbivora; man for the omnivora; and the dog or cat for the carnivora. In all cases throughout this work it is to be understood that, unless especially directed, the same tests are to be performed upon the urine of man and horse and a parallel record of such tests, for comparison, is to be kept upon the blank pages.

In man, fresh, normal urine is a clear, golden-colored, transparent liquid, having a peculiar aromatic characteristic odor, and a bitter saline taste.

In the horse, the urine is of a yellowish color when passed, but turns to a deep brown color upon standing for a time, due to the oxidation of pyrocatechin. It is more or less turbid and of a viscous character. Its odor is somewhat ammoniacal and strongly aromatic and more penetrating than that of man.

The urine is chiefly a solution of urea and certain organic and inorganic salts; epithelial cells and mucus may also be held in suspension. Like milk and other animal fluids, the urine is not of constant composition. It is influenced by various factors, such as food, the amount of water or other fluids taken into the body; the temperature of the skin; the emotions; blood pressure, general or local; exercise; the time of day; age; sex; and medicines.

Quantity. The amount varies considerably. In man, the quantity for twenty-four hours ranges from $1000 \mathrm{cc}$. to $2000 \mathrm{cc}$. In the horse, the average amount is about $3000 \mathrm{cc}$. to $4000 \mathrm{cc}$., although it may go as high as 7000 c.c. or $9000 \mathrm{cc}$. In the ox a still g:eater quantity is secreted, the usual limits being from $4500 \mathrm{cc}$. to $19000 \mathrm{cc}$. In the sheep, from $250 \mathrm{cc}$. to $700 \mathrm{cc}$. In 

the pig it varies from $1200 \mathrm{cc}$. to $6000 \mathrm{cc}$. In the dog it varies from $200 \mathrm{cc}$. to $900 \mathrm{cc}$., depending upon the size of the animal.

Color. The color ranges from pale yellow to brown. The normal color of urine is due to pigments probably derived from the coloring matter of the bile.

Transparency. The urine of the horse is normally more or less opaque, that of man should be transparent at the time of passing. Many pathological urines, however, are perfectly clear. Pathological turbidity may be due to urates, phosphates, pus, bacteria, spermatozoa, fatty globules, blood, etc. All urines become turbid after standing for a time.

The urine of the horse is especially turbid at the end of micturition, exceptionally it may be clear but becomes turbid on cooling. Its opacity is due to the presence of earthy salts of the carbonate of lime precipitated and formed by the disengagement of a certain amount of carbon dioxide from the bicarbonate of lime. The turbidity increases when the urine remains for any length of time in the bladder; it reaches its maximum when the urine is cooled by exposure to the free air; it diminishes after the ingestion of a large quantity of water.

A clear, limpid urine is generally pathological in the horse; it indicates polyuria and the reaction in this case is usually acid, exceptionally neutral or alkaline when the phosphates are modified qualitatively or quantitatively.

The turbidity of horse urine is abnormal when it is due to the presence of the phosphate of lime, or of calcium sulphate, or acid saits; also from the existence of albuminoid substances, (exudates, leucocytes in interstitial nephritis).

In other animals the passing of turbid urine is usually regarded as abnormal.

Consistency. In the horse the urine is very viscid on account of the contained mucus and sometimes of epithelial debris. It filters very slowly. Urine taken directly from the ureter or the pelvis of the kidney is still more viscid, having a consistency very similar to that of egg albumin and a specific gravity somewhat higher than normal.

Reaction. The reaction of human urine is acid, that of the dog more so than that of man. In the pig it is sometimes acid and sometimes"alkaline, depending upon the diet. In the horse 

and sheep it is alkaline, also in the ox, but in the calf and foal while suckling it is acid. Herbivorous urine is alkaline, but if such animals are starved for a time they practically become carnivorous in that they are living upon their own tissues and under such conditions their urine becomes acid.

In the herbivora the reaction is normally alkaline and is due to the presence of bicarbonates or carbonates of lime or potassium. It would appear that the salts present in the vegetable food undergo oxidation to form organic acids, and these in turn are transformed into bicarbonates or carbonates, causing alkalinity of the urine.

In man the acidity is due to the presence of acid sodium phosphate $\mathrm{NaH}_{2} \mathrm{PO}_{4}$. The urine passed before breakfast and during fasting or perspiration is more acid than at other times; during digestion and after meals the acidity is decreased.

The degree of acidity of the urine may be determined by the use of the acidimeter, a graduated glass tube devised by Dr. H. R. Harrower. His description of it and its use follows:

"The acidimeter consists of a glass tube so graduated that $10 \mathrm{cc}$. is the first measuring point. From this upward the tube is graduated in fifths of degrees up to $100^{\circ}$, each degree representing the amount of decinormal sodium hydroxide solution required to neutralize $100 \mathrm{cc}$. of urine. The method of using the acidimeter is as follows: The tube is filled with the urine to be tested, until the lower edge of the meniscus is just on the 10 cc. mark. Two drops of phenolphthalein indicator solution are added, and then with an ordinary medicine dropper decinormal sodium hydroxide solution is slowly added, inverting the tube after each addition, until the color of the fluid has just been changed from a yellow to a light rose pink. The acidity in degrees is now read off on the tube at the level of the fluid. The normal acidity of a mixed 24 hour specimen should be between 30 and 40 degrees.

(With very concentrated urines in which the acidity is above $100^{\circ}$ the tube may be filled to the $5 \mathrm{cc}$. mark and water to the usual level. The resulting figures are, of course, doubled.)

If the urine is alkaline in reaction and it is desired to estimate the degree of alkalinity decinormal hydrochloric or oxalic acid solution must be used in place of the sodium hydroxide, the pink color present being just discharged by the acid." 

The "Acid Index" or "Acid Unit" may be obtained by multiplying the degree of acidity by the amount of urine passed in 24 hours. The normal man is about 40,000 acid units.

In man there is increased acidity physiologically during the night; with a flesh diet; after strong muscular exertion; during the intervals of gastric digestion; after the ingestion of mineral acids.

There is increased acidity, pathologically, in fevers; in rheumatism; after asthmatic attacks; in emphysema, pneumonia and pleuritis.

The urine is less acid, or alkaline, physiologically, during gastric digestion; after hot or prolonged cold baths; after profuse sweating; after copious ingestion of vegetable acids and their salts.

Pathologically in acute and chronic inflammation of the urinary tract as in cystitis; in decomposition of the urine in the bladder in retention; in some cerebral and nervous diseases: in anemia; chlorosis; and general debility.

When the urine of man is set aside in a cool place it gradually becomes more acid. This is called acid fermentation. After longer exposure to a warm atmosphere the urine becomes neutral, and finally strongly alkaline in reaction. It becomes turbid, has an ammoniacal odor, and deposits triple phosphate, ammonium urate, and great numbers of microbes exist. This is alkaline fermentation and is due to the transformation of the urea into ammonium and carbon dioxide, by means of a ferment produced by an organism known as the micrococcus ureae. (Fig. 20). This organism is said to be conveyed through the air and to exist commonly around the orifice of the urethra. As long as the urine is acid the organism does not exist in the bladder, but may sometimes gain entrance through the medium of a sound or catheter.

Test the reaction of the urines with red and blue litmus paper. Some urines change both the red and blue paper and are termed amphoteric. (In taking notes of the experiments it is well to record the results in parallel columns, the horse urine in one column and the human urine in the other).

Specific Gravity. The specific gravity of human urine ranges from 1015 to 1025 , the average being 1018 to 1020 . That 

of the horse ranges from 1020 to 1050 , the average being about 1035. That of the cow is lower, ranging from 1015 to 1045 . It seems to depend considerably upon the milk secreted. In milch cows the urine contains a greater amount of water and a lesser amount of solids. The specific gravity of the urine of the sheep ranges from 1015 to 1060 ; that of the pig from 1005 to 1025 , and of the dog from 1016 to 1060 , depending upon the diet. Cat, 1020 to 1040 .

The specific gravity may be obtained in different ways. The simplest and most usual way is to employ the instrument known as the urinometer. Some urinometers are not strictly accurate, but they may be tested by filling the urinometer jar with distilled water at $15^{\circ} \mathrm{C}$. $\left(60^{\circ} \mathrm{F}\right.$.) Read the division of the scale corresponding with the surface of the fluid looking above or below the meniscus as is found to be the most correct for the zero reading. Always adhere to this method when using the same urinometer. Test the specific gravity of the urines and make the necessary corrections. If the urine is warmer than $15^{\circ} \mathrm{C}$. add 1 to the last right hand figure of the specific gravity for every 4 degrees of $\mathrm{C}$. temperature, or for every 7 degrees of extra F. temperature. As opportunity presents, test the specific gravity of some warm, freshly passed urine. Test the same urine later, when cool, and note if any difference in the reading.

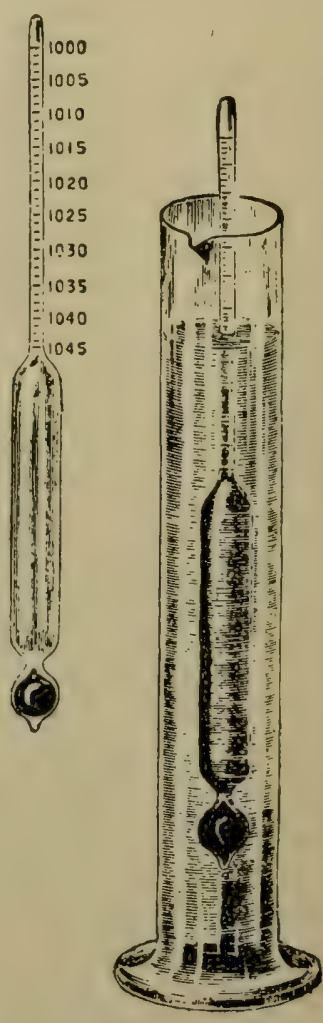

FIG. 3

Urinometer.

Urinometers already corrected for the ordinary room temperature $\left(70^{\circ} \mathrm{F}\right.$.) may be obtained, in which case the temperature corrections may be omitted. If the urine should be too dense to read easily on the urinometer, dilute it with an equal volume of distilled water and multiply the reading by two to 

get the correct specific gravity. The variation of the specific gravity depends upon the amount of the solids in the urine. The amount of solids may be estimated with approximate accuracy from the specific gravity by Christison's formula (Häser-Trapp's coefficient): "Multiply the last two figures of a specific gravity expressed in four figures by 2.33 . This gives the quantity of solid matter in every 1000 parts." (The number of grams in 1000 cc.).

Example. Suppose a patient passes $1400 \mathrm{cc}$. of urine in 24 hours and the specific gravity is $1020,20 \times 2.33=46.6$ grams of solids in $1000 \mathrm{cc}$. To ascertain the amount in $1400 \mathrm{cc}$. use the following proportion: $1000 \mathrm{cc}$ : $1400 \mathrm{cc}$. : : 46.6 grams is to $\mathrm{X}$ (65.24 grams). The total quantity of the solids of the human urine is about 60 grams for the 24 hours or approximately $4 \%$.

The following method taken from the Alkalcidal Clinic may also be used: Multiply the quantity, in ounces, of the 24-hour urine by the last two figures of the specific gravity and this by 1.1 , the product will represent the total solids in grains. Thus, if the amount of urine voided in 24 hours be 36 ounces and its specific gravity 1021 , the formula would be $36 \times 21 \times 1.1$, equal to 831 grains, the normal amount for a person weighing $100 \mathrm{lbs}$. The amount for other weights may be determined by proportion.

In general the amount of total solids is a measure (a) of the activity of tissue change; (b) of renal integrity; (c) of abnormal constituents in the urine. Hygienic conditions which favor increased metabolism, as abundant food, active exercise, etc., increase the solid matter in urine, while the opposite conditions decrease them.

.With the urine normal or subnormal in amounts, the solids are deficient pathologically from defecivve and enfeebled metabolism as in senility; anemia as a result of syphilis, cancer, etc.; chronic alcoholism; functional or organic diseases of the liver; from renal failure as in acute nephritis; certain conditions of chronic renal disease; at the close of Bright's disease; venous congestion of the kidneys, etc. With the urine increased in amount there may be a deficiency of solids in diabetes insipidus; interstitial nephritis; amyloid disease of the kidney; chronic parenchymatous nephritis. When the urine is not increased in amount, the urinary solids are increased in fevers; lithemia; some 

forms of dyspepsia. When the quantity of urine is increased the solids are increased in diabetes mellitus; phosphaturia; azoturia (excessive secretion of urea).

Calculate the solids of the urines by the formulae previously given. After obtaining the result of $1000 \mathrm{cc}$. estimate the quantity in $1250 \mathrm{cc}$. of human, and $5450 \mathrm{cc}$. of horse urine. In recording the tests use parallel columns, one column for the horse and the other for the human urine.

\section{III.}

Qualitative Tests. In all cases the urines must be filtered and perfectly clear before attempting the examination.

\section{INORGANIC CONSTITUENTS.}

These consist chiefly of sodium, potassium, ammonium, calcium, and magnesium, combined with hydrochloric, phosphoric and sulphuric acids.

Water. The water of the urine is derived from the food and drink, a small quantity being formed in the body. It varies according to the activity of the sweat glands of the skin.

Chlorides. Next to the urea the chlorides form the chief portion of the urinary solids. The chlorides are increased physiologically after the ingestion of salt foods and much water; mental and physical activities; and during pregnancy. Pathologically, they may increase after the crises of fevers; after the absorption of exudates; in diabetes (occasionally).

The chlorides are decreased pathologically, in all acute fevers; pneumonia (often entirely absent during the height of the disease); in cholera; and in most chronic diseases. An increase, or the re-establishment of the excretion of chlorides in disease is generally a favorable sign. In pneumonia it is a precursor of the crisis, and may often take place before other symptoms reveal the favorable change.

Test a portion of each urine with a few drops of silver nitrate solution. A white, cheesy or curdy precipitate 

insoluble in nitric acid indicates the presence of silver chloride. The phosphate of silver may also be thrown down but the nitric acid dissolves it, keeping it in solution.

Evaporate carefully a few drops of urine upon a glass slide, with a gentle heat over the flame. Octahedral or rhombic crystals may form,- - a compound of sodium chloride and urea. Examine with the microscope. (Fig. 5).

Sulphates. The sulphates are chiefly those of sodium and potassium. Only a small amount of them enters the body with the food, so that they are chiefly formed from the metabolism of proteids in the body. The above are known as ordinary sulphates. Another class known as the ethereal sulphates also exist. The proportion exists in the ratio of 10 of the ordinary to 1 of the ethereal in man. In the horse the proportion is about 2 of the ethereal to 1 of the ordinary. The ethereal sulphates are formed by the combination of sulphuric acid with organic bases such as phenol, skatol, etc., which originate from putrefactive processes in the intestine. The amount of ethereal sulphates is of importance in determining whether or not the digestive processes are going on normally. In general the sulphates are increased physiologically by the ingestion of sulphur and its compounds; nitrogenous food; and conditions of increased metabolism.

After acidulating the urine with hydrochloric acid to prevent the precipitation of phosphates, add to a small part of each urine, a little $2 \%$ barium chloride solution; a precipitate of barium sulphate is formed, insoluble in nitric acid.

To separate the ethereal sulphates, mix $30 \mathrm{cc}$. of urine with an equal bulk of "baryta" mixture. Stir and filter. This removes the ordinary sulphates (as barium sulphate), add $10 \mathrm{cc}$. of hydrochloric acid to the above filtrate, and keep in the water bath at $100^{\circ} \mathrm{C}$. for an hour in the hood and then allow the ethereal sulphates to settle. This may require some little time. (Baryta mixture is prepared by making saturated solutions in the cold, of barium nitrate and barium hydrate, and adding two volumes of the hydrate to one volume of the nitrate). 

Phosphates. The phosphates consist of alkaline and earthy salts in the proportion of 2 to 1 . The latter are insoluble in an alkaline medium and are precipitated when acid urine becomes alkaline. They are insoluble in water, but soluble in acids; in acid urine they are held in solution by free $\mathrm{CO}_{2}$. The alkaline phosphates (sodium and potassium) are very soluble in water, and they never form urinary deposits. The earthy are phosphates of calcium $\left(\mathrm{Ca}_{3} \mathrm{PO}_{4}\right)_{2}$ (abundant) and magnesium $\left(\mathrm{MgHPO}_{4}\right.$ plus $7 \mathrm{H}_{2} \mathrm{O}$ ) (scanty). An alkaline medium precipitates them although not in the form in which they occur in the urine.

The excretion of phosphates in the urine is largely dependent upon the amount of calcium ingested; the more calcium the food contains, the less phosphoric acid appears in the urine, and the more in the feces. This is due on the one hand to the tendency on the part of calcium to form insoluble calcium phosphates in the intestinal tract and in this way to prevent the absorption of the food phosphates; on the other hand, to the well-established tendency of calcium salts to be excreted into the bowel and not into the bladder; one must imagine in the latter case that calcium salts circulating in the blood combine with circulating phosphoric acid and bear the latter with them into the bowel.

The fact is of some therapeutic importance in the treatment of nephrolithiasis due to uric acid calculi, for the administration of calcium salts in this affection by bearing much phosphoric acid into the bowel, leads to the excretion of less phosphoric acid in the urine, and hence of normal and basic instead of acid phosphates; and as the latter precipitate and the former dissolve uric acid, it will be seen that by giving calcium we prevent the precipitation of crystalline uric acid and urate deposits in the urinary passages. (Croftan).

Where considerable calcium is present in the food the excretion of phosphates in the urine is minimum, especially if the urine is alkaline because of the presence of sodium or potassium salts. In herbivorous animals where the urine is alkaline and where considerable quantities of phosphorus containing food are eaten, very little phosphate is excreted in the urine.

Phosphates are increased in the urine pathologically in rickets; osteomalacin; osteoporosis; fractures; chronic rheumatism; diseases of the nervous system; and after great mental 

strain and worry. Phosphates are decreased in renal diseases and phthisis.

Physiologically, variations occur chiefly from the character of the food and drink; in the horse there may be an increase in the urinary phosphates after a large feed of oats, bran, oilcake, etc. Pathologically, they are increased during the active changes in such bone diseases as spavin, ring-bone, splint, etc.

To a small amount of each urine add about half its volume of nitric acid and then add two volumes of a $5 \%$ solution of ammonium 'molybdate and boil. A canary yellow ppt. (crystalline) of ammonium phospho-molybdate should appear in the omnivorous urine. In the herbivorous urine the presence of so much organic matter renders the test unreliable; although a precipitate may form it is not a typical or characteristic one for phosphates in the urine of the horse, unless the organic matter has been previously removed.

To each of the urines add half its volume of ammonia and allow it to stand. A precipitate of earthy phosphates is formed, in the urine of man. Filter, add enough nitric acid to give an acid reaction to the urines, and test the filtrates with ammonium molybdate as before. This method separates the earthy from the alkaline phosphates.

To a portion of the urines add half their volumes of baryta mixture; a copious precipitate. Filter, add nitric acid and test the filtrates with ammonium molybdate. No ppt. should occur as the baryta mixture precipitates the phosphates as well as the sulphates and carbonates.

Use a little of the magnesia mixture instead of the baryta mixture. Filter, add a little nitric acid, and test the filtrate with ammonium molybdate. (The magnesia mixture is composed of magnesium sulphate 1 part, ammonium chloride 1 part, ammonia water 1 part, and distilled water 8 parts).

To portions of the urines add a few drops of acetic acid and then a little $5 \%$ uranium nitrate solution,--a yellow ppt. of uranium phosphate is formed.

The lime, magnesia, iron and other inorganic urinary constituents are comparatively unimportant, and have no special 

clinical significance. The tests for them are somewhat complicated and are therefore omitted.

Demonstration of Carbonates and $\mathrm{CO}_{2}$ in Urine. Carbon dioxide exists in the urine to some extent in a free state. There are also various carbonates present, especially in the herbivorous urine. The amount is very variable and to a great extent is dependent on the kind of food that is eaten; large quantities of vegetable foods determine an increase both in the combined and in the free carbon dioxide. The carbonates may be broken up and $\mathrm{CO}_{2}$ given off by the application of heat or certain acids.

Heat experiment. Fill a small flask about half full of unfiltered herbivorous urine. Through the perforated stopper of the flask pass some bent glass tubing connected with a test tube containing lime water. Heat the urine in the flask, and as it boils the $\mathrm{CO}_{2}$ will pass over into the test tube, and calcium carbonate will be formed from the union of the gas with the lime. Repeat the experiment with omnivorous urine.

- A simple proof of the presence of carbonates, or $\mathrm{CO}_{2}$ in the urine is to fill a Doremus Ureometer with $25 \mathrm{cc}$. of the unfiltered urine and introduce $1 \mathrm{cc}$. of nitric acid. The larger part of the gas $\left(\mathrm{CO}_{2}\right)$ rises and collects in the upper portion of the ureometer. Compare the amounts thus obtained in the urines of horse and man.

A simpler qualitative test is to add a few drops of nitric or acetic acid to a little unfiltered urine in a test tube. If effervescence occurs it is due to $\mathrm{CO}_{2}$ set free from the carbonates by the acid. 



\section{IV.}

\section{ORGANIC CONSTITUENTS.}

Urea is, in amount, the principal constituent of the solids of the urine. It is the most important product of the decomposition of proteid in the food. In round numbers it forms from $2 \%$ to $3.5 \%$ of the urine, averaging about $2.5 \%$. About one-half of the total solids in the urine consists of urea. Urea has no effect on litmus, it is odorless, has a weakly cool and bitter taste like saltpeter. It is very soluble in water amd alcohol, but it is almost insoluble in ether and benzine. About $90 \%$ of the total nitrogen of the urine is excreted in the form of urea.

Physiologically, urea is increased by a proteid diet; exercise and muscular vigor; by drinking much water. It is decreased by fasting; non-nitrogenous food; reduction of water in the diet; alcoholic beverages, tea or coffee; indolence of mind and body. Pathologically, it is increased in all acute fevers; dyspnoea; diabetes; and phosphorus poisoning. It is decreased in uremia; acute yellow atrophy of the liver and in chronic diseases. In general, any disease that interferes with the activity of the liver decreases the urea. Any disease affecting the uriniferous tubules may modify the appropriation of the urea from the blood and affect its passage into the urine. An increase of the urea independently of the physiologic variations and amount of nitrogenous food eaten is an approximate index of the amount of tissue waste in the system; on the other hand, when the urea is decreased it is an evidence of a diseased condition of the liver (the producer of urea) or the kidney (the eliminator of urea).

A simple method of detecting urea is to concentrate a small amount of dog* or human urine in an evaporating dish to about half of its original volume. Place a drop or two of this concentrated urine upon a glass slide and after adding a drop of nitric acid, gently warm over the flame. If urea be present, upon evaporation, the microscope will show the characteristic crystals of nitrate of urea, of rhombic or hexagonal form. (Fig. 4).

*For this experiment, dog urine is more satisfactory than human because of the larger percentage of urea present. 

Take $20 \mathrm{cc}$. of fresh, filtered dog or human urine and add $20 \mathrm{cc}$. of baryta mixture to precipitate the phosphates and sulphates. Filter, evaporate the filtrate to dryness, and extract the residue with a little boiling alcohol over the water bath very carefully. Filter off the alcoholic solution, stand the filtrate away in a cold place for crystallization. The crystals of urea, usually long, fine, transparent needles, will separate out. Examine them under the microscope. (Fig. 5).

Repeat in the hood, the experiment upon the urine of the horse and compare results.

Heat some urea crystals in a test tube. Biuret is forméd and ammonia comes off. Add a trace of copper sulphate solution and a few drops of $20 \%$ caustic potash. A rose-red color is produced,- - the biuret reaction.

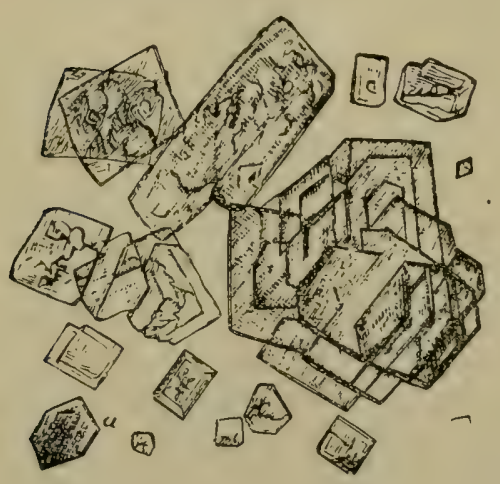

FIG, 4

Crystals of Nitrate of Urea.

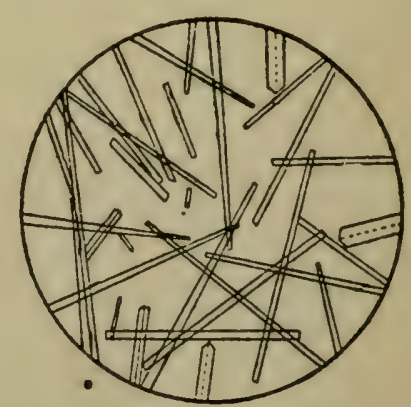

FIG. 5

Crystals of Urea.

Medicines which increase the amount of urea are: urea itself; uric acid, common salt, phosphoric acid, squill, theobromine, colchicum, cubebs, atropine, cantharides, vegetable acids, iron preparations, hyposulphite of soda, potassium chloride, ammonium chloride, coca, potassium permanganate, oxygen, salicylic acid.

Medicines which decrease the amount of urea are: digitalis, alcohol, coffee, tea, potassium and sodium iodides, potassium bromide, arsenic, turpentine, aklaline carbonates, mercury, antipyrin, valerian, quinine sulphate, benzoic acid. 

Uric Acid, sometimes called lithic acid, is, next to urea, the most important nitrogenous constituent of the urine of man. It has been said to be absent in herbivorous urine, being replaced by hippuric. This has been shown by later researches not to be strictly true, as a trace of uric acid is found in addition to the hippuric. In birds and reptiles uric acid is the chief nitrogenous constituent, being present in greater amount than urea.

In man the proportion of urea to uric acid is about 45 to 1 , about 0.5 gram of the latter being excreted in the 24 hours. It causes the brick red deposit sorretime seen in urine after standing for a time. Its solubility is very low, only 1 part being soluble in $14000 \mathrm{cc}$. of cold water, and 1 to 18000 in boiling. Physiologically, it is increased and diminished proportionately with urea. Pathologically, it is increased in indigestion; acute dropsies, rheumatic and catarrhal inflammations; after attacks of gout; cancer of the liver; in leucemia; in all disturbances of the circulation and respiration. Pathologically uric acid is decreased in chronic diseases generally; diabetes and polyuria; before paroxysms of gout; anemias; chronic rheumatism; chronic diseases of spinal cord.

Uric acid is generally in solution in the form of urates of sodium, potassium, ammonium, lime and magnesium. These salts are very easily decomposed even by weak organic acids.

Perform the following tests upon both urines (filtered).

In a conical glass, add 5 parts of hydrochloric acid to 30 parts of urine. Label and put in a cool place for 24 hours. Red or brownish colored crystals of uric acid are deposited upon the sides of the glass, or form a pellicle on the surface of the fluid like fine grains of cayenne pepper. The brownish-red color is due to pigment (uroerythrin).

Murexide Test. To about $1 \mathrm{cc}$. of human urine add a little nitric acid; evaporate in a porcelain dish very carefully to avoid charring. Cool and add a drop of dilute ammonia, a purple red color of murexide or purpurate of ammonia is formed. It turns bluer upon the addition of caustic potash solution.

Dissolve a few crystals of uric acid in $10 \%$ caustic soda or potash. Add a drop or two of Fehling's Solutionor dilute cupric sulphate and caustic potash and heat- 

there should occur a ppt. which at first may be white and after a time turning green or reddish.

(Fehling's Solution is put up in two bottles; one labeled $\mathrm{A}$, the other $\mathrm{B}$. In making tests, take equal parts of $\mathrm{A}$ and $\mathrm{B}$ and add the substance to be tested. See formula in appendix.)

Schiff's Test. Dissolve a little uric acid in a small quantity of $10 \%$ sodium carbonate solution. With a glass rod, place a drop of silver nitrate solution on filter paper and then a drop of the uric acid solution so that the two drops partially overlap. A dark brown or black spot of reducing silver appears.

Hippuric Acid, $\left(\mathrm{C}_{9} \mathrm{H}_{9} \mathrm{NO}_{3}\right)$, is found especially in the urine of the herbivora, as the horse, ox, etc. In the urine of carnivora, and especially in that of man, it exists in but very minute quantity, usually about 0.5 to 1 gram being excreted in 24 hours. It dissolves readily in hot alcohol but is sparingly soluble in water. It occurs in man after the ingestion of certain vegetables, such as asparagus, plums, pears, and apples with their skins; a purely vegetable diet and from the use of benzoic acid; cinnamic acid, essence of bitter almonds, quinine, and analogous bodies. Hippuric acid normally seems to be derived chiefly from the husks or cuticular structures of the food.

Pathologically hippuric acid is increased in diabetes, chorea; jaundice and other liver complaints; and in the acid urine of

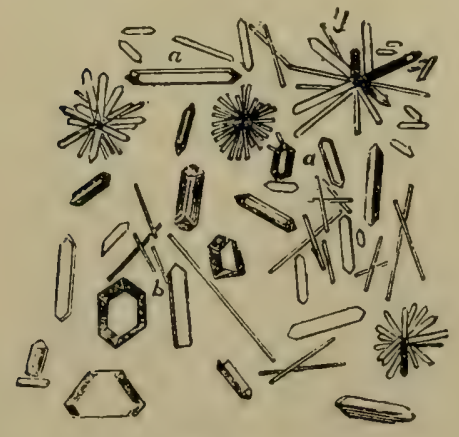

FIG. 6

Crystals of Hippuric Acid.

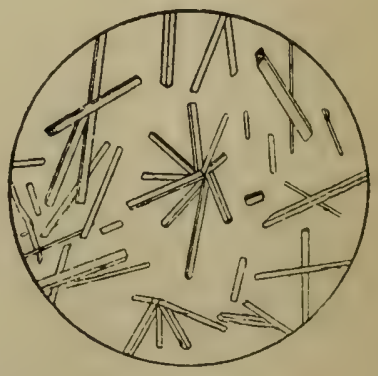

FIG. 7

Various forms of Hippuric Acid with triple phosphates. 

patients suffering from all kinds of fevers. In testing for hippuric acid the fresh urine should be used; if stale, benzoic acid is likely to be obtained instead.

Test. Saturate the fresh urine with lime water, which transforms the hippuric acid into a salt of lime, the fluid is boiled, then filtered, evaporated to a syrupy consistency, cooled and excess of hydrochloric acid added, when hippuric acid crystallizes out on standing for 24 hours. The horse urine is to be evaporated in the hood.

Creatinin $\left(\mathrm{C}_{4} \mathrm{H}_{7} \mathrm{~N}_{3} \mathrm{O}\right)$. This substance was discovered in - the urine by Liebig. It is easily produced from creatin, a substance normally existing in plain and striated muscular tissue. Creatinin occurs constantly in normal human urine, the amount varying according to Voit, from 0.5 to 4.9 grams per day according to the quantity of proteids eaten. It is said not to be diminished by fasting.

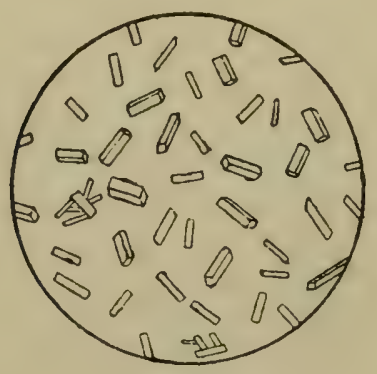

FIG. 8

Creatinin.

Pathologically it is increased in typhoid fever; intermittent fever; pneumonia; tetanus. It is decreased during convalescence from the above diseases; and likewise in anemia; chlorosis; muscular atrophy; tuberculosis; paralysis, etc.

Test. Take 5 cc. of urine in a test tube and add a few drops of freshly prepared $1 \%$ sodium nitroprusside. Render the solution alkaline with potassium hydroxide. A ruby red color develops which soon turns yellow. The above is Weyl's test. Acetone, if present, also gives the red reaction.

Perform the above test on both urines.

Caution. Creatinin reduces copper oxide and may be taken for small quantities of sugar.

Mucus. Mucus in the urine is not visible, but causes cloudiness sometimes by entangling epithelial cells, urates, oxalate of lime and other crystals in various amounts.

Add to the urine a little acetic or citric acid and, in addition a few drops of liqucr iodi comp. (Lugol's solu- 

tion) which makes the threads or bands of mucin visible.

Indican or Indoxyl. This substance is derived from indol, one of the putrefactive products formed in the intestine. Indoxyl occurs in very small quantities in normal human urine, about .004 to .020 gram for the 24 hours. Horse's urine is said to contain 23 times as much. The intestines of the herbivora are much longer than in the case of the carnivora. On this account, and in conjunction with the carbohydrate diet, a much greater fermentation occurs, which leads to a greater elimination of indican in the urine.

In obstruction of the intestine, or in intestinal catarrh or where the food remains a long time in the intestine and ferments there, the proportion of indican increases in the urine and causes a true indicanuria. Indoxyl is of considerable clinical importance, an increase is indicative of imperfect performance of the digestive processes. In obstructive diseases of the small intestine the increase of indoxyl in the urine is enormous.

Pathologically indoxyl is increased in cholera, typhoid fever, peritonitis, dysentery, Addison's disease, cancer of the liver and stomach and pernicious anemia.

Obermayer's Reagent. This reagent has the advantage of keeping indefinitely. It is prepared by dissolving 2 grams of solid ferric chloride in $500 \mathrm{cc}$. of concentrated hydrochloric acid. (Sp. gr. 1.19).

To equal parts of urine and the above reagent add a little chloroform. Shake frequently but not too violently (otherwise an emulsion may be formed). The chloroform will become more or less blue by the indigo formed, in proportion to the indican originally present: According to the depth of the blue color it may be designated as little, much or copious.

A test said to be somewhat more sensitive is as follows: Urine 10 cc.; $20 \%$ basic Lead acetate 2 cc. Shake and filter. Add to the filtered urine $10 \%$ Thymol in alcohol, $1 / 2$ cc.; Obermayer's Reagent $10 \mathrm{cc}$. and let stand for 15 minutes; add chloroform, $4 \mathrm{cc}$. Shake gently several times. Let the chloroform settle. A violet color indicates the presence of indican.

Jaffe's Test. To a little urine add an equal volume of strong hydrochloric acid. Add to this mixture 2 or 3 

drops of a solution of freshly prepared chlorinated soda. There soon forms a bluish cloud of indigo. Add a little chloroform and shake gently, this will take the indigo into solution and settle as a blue layer at the bottom of the test tube. The amount of indoxyl can be judged by the depth of the blue color. Indican is oxidized by free chlorine obtained from the chlorinated soda to indigo.

Oxalic Acid. This is usually found in combination with lime in the form of calcium oxalate. The crystals are of small. size and appear in the form of dumb bells and octahedra. They occur normally in greater amount in herbivorous than in omnivorous urine. They greatly increase after eating such vegetables as tomatoes, fresh beans, beet-root, asparagus, apples, grapes, honey, and after the use of rhubarb, senna, squills, etc. Another source of oxalic acid in the body is incomplete oxidation of carbohydrates and proteids or retarded metabolism. It is therefore a result of mal-assimilation and is found in dyspepsia, diabetes mellitus, etc. The long continued excretion of an excess of oxalate of lime frequently irritates the kidneys, producing albuminuria and grave nervous disturbances and may lead to the formation of calculi. (Fig. 18.)

Acetone. Normal urine may contain traces of acetone but it occurs in excessive quantities as a pathological condition.* It is found in many of the fevers, certain forms of cancer, in starvation, and in diabetes, when it indicates an advanced form of the disease. It is associated with an increased proteid metabolism and is looked upon as a product of proteid decomposition with deficient oxidation.

Lieben-Ralfe Test. Dissolve 1.3 grams (20 grains) of potassium iodide in $4 \mathrm{cc}$. (1 dram of liquor potassae). Boil in test tube, after which gently pour the urine on its surface. A yellow precipitate between the two solutions indicates an affirmative test. A more satisfactory test is to add to the urine a few crystals of iodine and of iodide of potassium with some caustic potash. Heat. Yellow precipitate-iodoform with its characteristic odor.

Legal's Test for Acetone. Add to $5 \mathrm{cc}$. of the urine

*If pathological urine is not available, a small amount of acstone may be added to the urine for laboratory tests. 

some fresh aqueous solution of sodium nitroprusside followed by a little ammonia, or sodium hydrate solution, which gives a red color. Add an excess of acetic acid and if acetone is present the red color will be intensified, if acetone is absent a yellow color will result. Compare with creatinin.

These tests are not always satisfactory when applied to the ordinary urine. Greater accuracy is claimed if the urine is distilled and the tests applied to the distillate.

Frommer's Test for Acetone. To $10 \mathrm{cc}$. of urine in a test tube add some potassium hydroxide to make the urine markedly alkaline; before the latter is dissolved add 10 to 12 drops of salicylic aldehyde (made by dissolving 1 part of salicylous acid in 10 parts of absolute alcohol). Heat the mixture to about $70^{\circ} \mathrm{C}$. With acetone the solution becomes yellow, then red and after long standing dark red. According to Frommer, even the minutest amount of acetone will give this reaction and no other constituent of the urine will give this color-not even diacetic acid. The reaction is explained as follows: One molecule of salicyclic aldehyde combines with one molecule of acetone to form oxybenzol-acetone. This, in the presence of strong alkalies, forms dioxy-dibenzolacetone. The alkaline salts of this compound are intensely red.

Diacetic Acid. Lindemann's modification of Riegler's test.

Urine

Acetic Acid

Lugol's Solution

Chloroform
$10 \mathrm{cc}$.

5 drops

5 drops

$3 \mathrm{cc}$.

With normal urine the chloroform is colored rosy red; with urine which contains diacetic acid it remains colorless. Urine which contains much uric acid should have the amount of Lugol's Solution doubled, and should not be too vigorously shaken.

Urobilin is commonly regarded as the most important coloring matter in the urine. There is some evidence that it represents a reduced form of bilirubin, one of the pigments of the bile.* Urobilin is more readily obtained from highly colored urines, (fevers, etc.):

*A small amount of an alcoholic extract of the feces added to the urine will usually give favorable tests for urobilin. 

Test. The ordinary test with an alcoholic solution of zinc may be simplified in the following manner: $10 \mathrm{cc}$. of urine are acidified with 2 drops of $\mathrm{HCl}$ and shaken with 2 cc. of chloroform. After the separation of the liquids, $2 \mathrm{cc}$. of the chloroform layer are tested with $4 \mathrm{cc}$. of a solution of 1 gram of crystallized acetate of zinc in a liter of $95 \%$ alcohol (shelf reagent). At the junction of the two layers the green fluorescent ring characteristic of urobilin will appear; and on shaking, a fluorescence which is rose-colored by reflected light will be distinguished throughout the liquid.

Another test is to add ammonia to the urine until distinctly alkaline, filter, and to the filtrate add a little $10 \%$ chloride of zinc solution. A green fluorescence should appear, and if examined with the spectroscope, a characteristic band should occur. (See Fig. 16.)

Leucin and Tyrosin are pathologic constituents of urine. They are normal products of pancreatic digestion and under ordinary conditions are carried, after absorption, to the liver, where they disappear, presently undergoing decomposition. When present in the urine these bodies are usually considered pathognomonic of acute yellow atrophy of the liver, although they are likewise stated to be present in the urine in certain rare cases of acute phosphorus poisoning associated with hepatic atrophy, due to typhoid fever, etc.

Phenol. According to Tereg and Munk the horse excretes in the urine about 10 grams of tribromphenol in 24 hours. The tribromphenol is equivalent to 3 grams of phenol daily. Great importance is laid by these observers on the excretion of phenol, a process which is suspended during intestinal complaints, particularly colic, and is, according to them and others, a cause of the rapid death in these effections, produced by the toxic effect of the unexcreted phenol. The production of phenol in the healthy body is greatly influenced by diet, being largest on rye and hay, one part peas and two parts oats, and on hay alone; it is smallest on rye alone, and next smallest on oats and hay. Salkowski is inclined to regard the excretion of 3 grams of phenol daily as too high. 

V.

\section{ABNORMAL SUBSTANCES FOUND IN THE URINE.}

Albumin. The presence of this substance in the urine is regarded as pathologic. There is, however, in the urine of some individuals apparently enjoying perfect health, minute traces of albumin sometimes present, and, unless these traces persist, are not to be regarded as serious. If present in any considerable quantity, it must be regarded as distinctly abnormal. Albuminuria is the term applied when albumin occurs in notable quantity in the urine. The principal form of albumin present is serumalbumin, in addition there may be serum-globulin, acid albumin, albumose, and peptone.

The amount of albumin in the urine may be increased: 1 . By food rich in albumin; 2. Suppression of cutaneous perspiration, as by colds, burns, or cutaneous diseases; 3. Pulmonary and cardiac diseases attended with dyspnoea, cyanosis, valvular diseases of the heart; 4. Febrile and inflammatory diseases, as malarial, eruptive, typhus and typhoid fevers, croup, diptheria, erysipelas, rheumatism, gout, peritonitis, meningitis, etc.; $5 . \mathrm{By}$ lesions or prostration of the nervous system, especially when attended by diminished temperature and arterial tension, as from grief, fear, injury, pressure; 6. Pressure as from tumors, pregnancy, etc.; 7. Cachexias, as from cancer, syphilis, scrofula, septicemia; 8 . Hydremia and ailments that disturb the vascular tension; 9. Chorea, convulsions, exacerbations of febrile and other diseases; 10. Diseases of genito-urinary organs, as Bright's disease, cystitis, hemorrhage, abscess, etc.; 11. Medicines, such as copaiba, cubebs, turpentine, some emetics and drastic cathartics, some anesthetics, coffee, many metallic salts, poisoning by hydrogen arsenide, carbon protoxide, carbon dioxide, phosphorus, iodine, iodoform, etc.

The loss of organic material (albumin) disturbs nutrition, the blood becomes more aqueous; it sometimes produces an anasarca which is the result of hydremia and of anuria.

Albuminuria may be caused: 1 . By an alteration in the renal transudation; 2. By certain changes in the blood; 3. By disturbance of the circulation. 

Renal Changes. Lesions of the dialysing portions of the kidney, especially of the glomerule; the epithelium of the convoluted tubules may also be essential in the production of albuminuria. These cells normally prevent the albumin from filtering through with the other elements of the plasma. Albuminuria is also dependent upon renal lesions; sometimes primary as in nephritis, sometimes consecutive as in alteration in the blood or a disturbance in the circulation. Acute nephritis, chronic nephritis, fatty or amyloid degeneration interfere with the process of dialysis.

Bacteria may exercise a traumatic action upon the renal epithelium and cause desquamation or degeneration, obstruct the vessels, modify blood pressure, or by the excretion of soluble products irritate the parts.

Changes in the Blood. The dialysing membrane cannot stand with impunity any adulteration of the blood. The passage, in the kidney, of any such substance as biliary pigment in icterus; glucose in diabetes; poisons, such as alcohol, lead or mercury, or toxic gases, render the urine albuminous.

Subcutaneous injections of solutions of extractives (leucin, tyrosin, creatinin, xanthin and hypoxanthin) cause degeneration of the epithelium of the kidneys and albuminuria. The subcutaneous injection of tincture of cantharides causes, in a few minutes, the production of an albuminous exudate in the glomerules. Although the limit of saturation of the blood plasma by albumin may be unknown, it is none the less evident that a superabundance of the substance (albumin) in the vessels causes albuminuria.

The existence of a physiologic albuminuria is still doubtful in the domestic animals; for Fröhner, who has examined the urine of a number of healthy horses, has found only two cases in which albumin was present. In man it has been demonstrated to be due to severe muscular exercise, slight cold, and nitrogenous diet.

Disturbance of Circulation. Too great a variation in blood pressure will cause albuminuria.

Renal emboli, section of vaso-motor nerves of the kidney, medullary lesions, etc., cause albuminuria by causing an active congestion of the kidney. Venous stasis, organic affection of the heart, of the liver, presence of fetus, etc., cause albuminous urine.

The urine may be less fluid. Bacteria may frequently cause 

thromboses, emboli, edemas, anemias, etc., from vaso-motor troubles changing simultaneously the filter, the liquids which filter, with regard to pressure and velocity.

Albuminous urine is usually of light color and low specific gravity. It may occasionally be dark and dense, due to other ingredients, or to concentration.

Under particular conditions of fatigue or disease albumin may appear in the urine.

Temporary albuminuria is sometimes induced by a cold bath, especially in persons prone to kidney disease, and it has been observed after excessive muscular exercise, as in the urine of soldiers after a prolonged march.

Any cause which leads to an increased blood pressure in the kidneys tends to induce albuminuria, and many of the cases in which it is the result of disease may be traced to this cause. Albuminuria is a constant accompaniment of the nephritis following scarlet fever and may occur to a less extent in pneumonia, typhoid and diptheria. It may also occur in diabetes, and is then a highly unfavorable symptom.

In every case the urine must be clear before testing, by filtering it carefully; also take the specific gravity.* In addition to the suspected urines make control tests on the normal for comparison.

Heat Test. Heat about $5 \mathrm{cc}$. of the urine to the boiling point in a test tube. Note the slightest turbidity. If present it will be due to albumin or earthy phosphates. In horse urine the precipitate may be due to driving off $\mathrm{CO}_{2}$ and precipitation of lime, etc., not phosphates. Add slowly a few drops of acetic acid (or nitric). If due to the phosphates the urine becomes clear, if the turbidity remains it is albumin. Care must be taken, in the addition of the acid after boiling, to note the effect after each drop is added and to go on adding until there is no doubt that the urine is distinctly acid. If only a trace of albumin is present and too much acid is added the albumin may be converted into acid albumin and remain in solution. Heat does not coagulate acid albumin. Add a little acetic acid to dissolve any phosphates and heat again.

*If a pathological urine is not available, a little blood serum added to the normal urine will give satisfactory tests. 

Another Heat Test. Fill a test tube about one-third full of water and boil it. Add a few drops of the suspected urine. If albumin is present a cloudiness or coagulum will appear, according to the amount of albumin present.

Heller's Cold Nitric Acid Test. Pour some of the urine gently upon the surface of some nitric acid in a test tube. A ring of white coagulum occurs at the junction of the two fluids. If the quantity of albumin is small, the coagulum may not occur for a few minutes. A brown zone will frequently be seen at the point of contact due to the action of the acid upon the coloring matters of the urine, but it does not give any turbidity unless albumin be present.

Millard-Robert's or Nitric Magnesian Test. The reagent is as follows: Nitric Acid, 1 part; Sat. Sol. Magnesium Sulphate, 5 parts. Use as in the preceding test.

Picric Acid Test. (Johnson's). Fill the test tube half full of urine. Slightly incline the tube and gently pour down its side about $2 \mathrm{cc}$. of a saturated solution of picric acid, so that it may come in contact with the upper layer of the urine. Place the tube in an upright position. A layer of coagulated albumin will appear at the line of junction. The coagulation of albumin takes place at once and is thus not easily mistaken for precipitated urates, which require some time for their precipitation and disappear on the application of heat.

Ferrocyanide Test: To $2 \mathrm{cc}$. of acetic acid in a test tube add $4 \mathrm{cc}$. of a $5 \%$ solution of potassium ferrocyanide. Mix them and add $10 \mathrm{cc}$. of urine. A precipitate will appear if albumin be present. No heat is required.

A test equally as good is that proposed by Zouchloss in which potassium sulphocyanide is substituted for the ferrocyanide.

Sugar in the Urine. Dextrose or glucose exists in the blood from 0.8 to 1.25 parts per 1000 . When a greater amount than 3 parts per 1000 exists, the excess is excreted through the kidneys. It is maintained by many that a trace of dextrose is normally present in the urine and may appear in slightly larger quantities transitorily without pathologic significance. The presence of small quantities of sugar in the urine is designated as glycosuria; 

in larger quantities it is known as diabetes mellitus. The former condition if habitual, is unnatural, and may terminate in the latter.

These diseased conditions do not necessarily point to diseases of the kidneys or urinary organs, but rather to the liver. The kidney, in ridding itself of this product (dextrose), becomes irritated, and this irritation extends down the entire canal, and we thus have a real polyuria produced.

Sugar, in sufficient quantity to react to ordinary tests, is found in the urine physiologically during pregnancy and lactation; in infants under two months old; in old persons living largely upon starchy and saccharine food. Pathologically in diabetes mellitus; in impeded respiration from pulmonary diseases; in impeded hepatic circulation (functional and organic diseases of the liver); in diseases of the central nervous system (general paresis, epilepsy, dementia, puncture of the fourth ventricle); in intermittent and typhoid fevers, by the action of certain poisons, as carbon monoxide, arsenic, chloroform and curare; in abnormally stout persons.

The persistent excretion of easily recognizable quantities of sugar constitutes diabetes. The quantity of urine is often enormously increased, as much as $10000 \mathrm{cc}$. being passed in 24 hours, by man. The specific gravity is high, varying from 1025 to 1050 . The color is usually pale, from the dilution - not diminution-of the urinary pigments.

The presence of albumin interferes with the tests for sugar and must, in all cases, be removed by the addition of acetic acid and heat. The urine, after being filtered, may then be used for the sugar tests.

The particular property of glucose, which is utilized for its detection, is its action as a reducing agent-its disposition to absorb oxygen. In this property it differs strikingly from saccharose or common cane sugar.

Principle of the Copper Tests. If a little copper sulphate and an excess of a solution of caustic potash be added to a solution of glucose, a clear blue solution results. Without the glucose, the alkali would precipitate the pale blue cupric hydrate, $\mathrm{CuO}_{2} \mathrm{H}_{2}$; and if the mixture were boiled this blue precipitate would be reduced to a black precipitate of Cupric Oxide, $\mathrm{CuO}_{2}$. The clear blue solution containing glucose, however, when boiled, changes from transparent blue to opaque yellow, and speedily 

deposits a yellow, ultimately red, precipitate of cuprous oxide $\mathrm{CuO}$. When the quantity of sugar is large the change is immediate. When small the reaction takes a few minutes for its completion.

Fehling's Solution. Solution A. 34.64 grams of pure crystalline copper sulphate are powdered and dissolved in $500 \mathrm{cc}$. of distilled water. Solution B. Sodio-potassium tartrate (Rochelle Salts) 173 grams. Pure caustic potash 125 grams. Add enough distilled water to make $500 \mathrm{cc}$. When using take equal parts of Solutions A and B. (If diluted with 5-10 vols. of water the test is said to be more sensitive).

Place some Fehling's Solution in a test tube and boil it. If no yellow discoloration takes place it is in good condition. Add a few drops of the suspected urine and boil. If the mixture suddenly turns to an opaque yellow or red color, the presence of a reducing sugar is indicated. Normal horse urine probably because of its pyrocatechin usually changes the color of the copper solution, but this does not indicate sugar.

Benedict's Modification of Fehling's Test. Greater delicacy and accuracy are claimed for this test. There are two solutions as in Fehling's. The first is prepared by dissolving 34.65 grams of cupric sulphate in a small anount of water and made up to $500 \mathrm{cc}$. The second by dissolving 100 grams of anhydrous sodium carbonate and 173 grams of Rochelle salt dissolved in water and made up to $500 \mathrm{cc}$. These solutions should be preserved separately in rubberstoppered bottles and mixed in equal volumes when needed for use. This is done to prevent deterioration.

To $2 \mathrm{cc}$ : of Benedict's solution in a test tube add $6 \mathrm{cc}$. of distilled water and not more than 7 to 9 drops of the urine under examination. Boil the mixture vigorously for 15 to 30 seconds and allow it to cool to the room temperature. If sugar is present in the solution a precipitate will form which is often bluish-green or green at first, especially if the percentage of sugar is low, and which usually becomes yellowish on standing. If the sugar present exceeds $0.06 \%$ this precipitate generally forms at or below the boiling point, whereas, if less than $0.06 \%$ of sugar is present the precipitate forms more slowly and generally only after the solution has cooled. 

Benedict has further modified the test by making a single solution which does not deteriorate upon long standing. The formula is as follows:

Cupric Sulphate

Sodium Citrate

Sodium Carbonate (anhydrous)

Distilled water to
17.3 grams

173.0 "

100.0

$1000.0 \mathrm{cc}$.

With the aid of heat the sodium citrate and carbonate are dissolved in about $600 \mathrm{cc}$. of water. Pour (through a folded filter if necessary) into a glass graduate and make up to $850 \mathrm{cc}$. The cupric sulphate is dissolved in about $100 \mathrm{cc}$. of water and made up to $150 \mathrm{cc}$. The carbonatecitrate solution is poured into a large beaker and the cupric sulphate solution is added slowly, with constant stirring. The mixed solution is ready for use, and does not deteriorate upon long standing.

The procedure is as follows: To $5 \mathrm{cc}$. of the reagent in a test tube add not more than 8 drops of the urine to be examined. The fluid is then boiled vigorously for one or two minutes and then allowed to cool spontaneously. In the presence of dextrose the entire body of the solution will be filled with a precipitate, which may be red, yellow or green in color, depending upon the amount of the sugar present. If no dextrose is present, the solution will either remain perfectly clear, or will show: a very faint turbidity, due to precipitated urates.

Trommer's Test. To 4 or $5 \mathrm{cc}$. of urine, in a test tube, add one-half its volume of $20 \%$ caustic potash and 2 or 3 drops of a solution of copper sulphate (1-10). Heat to the boiling point. If sugar be present a yellowish or reddish precipitate is thrown down, the sugar having reduced the cupric hydrate to cuprous oxide.

Bismuth Test. Put equal quantities of urine and $20 \%$ solution of caustic potash in a test tube and add a pinch of subnitrate of bismuth. Boil the mixture and if glucose be present the powder turns black. Albumin and sulphur also reduce bismuth and must be removed if the test is to be reliable. Bismuth is more or less blackened with normal horse urine. 

Nylander's Reagent. A solution is made of bismuth subnitrate 2 grams; Rochelle salts 4 grams; potassium hydroxide 10 grams; and distilled water to $100 \mathrm{cc}$. The urine is heated to boiling and a few drops of this alkaline solution of bismuth added, and on continuing the boiling, if sugar be present, the mixture turns black. The test is delicate, as little as $0.025 \%$ of glucose can be detected.

Phenylhydrazine Test. (Modified from Kowarsky). To 5 drops of pure phenylhydrazine and 10 drops of glacial acetic acid in a test tube is added 1 cc. of a $10 \%$ solution of sodium chloride. After shaking the mixture, $3 \mathrm{cc}$. of the urine are added and the test tube heated for two minutes or longer. The fluid is then allowed to cool slowly. If the sugar content exceed $0.5 \%$ the precipitate of glucosazone takes place in about two minutes. Small quantities of albumin do not hinder the reaction, but are precipitated by boiling. After an hour or more examine the precipitate microscopically. A $10 \%$ solution of sodium hydroxide has been recommended as a substitute for the sodium chloride solution and greater delicacy is claimed for the reaction.

The glucosazone crystals will have the form typical of skeins of wool, which will generally be double, whereas other crystals precipitated, such as those of glycuronic acid, are irregularly formed. As little as $0.005 \%$ (onefortieth grain per ounce) of glucose can be detected in urine by the phenylhydrazine reaction.

Uric acid, urea, xanthine, and creatinin in no way stimulate the reaction of glucose with phenylhydrazine. The only bodies which can offer confusion are glycuronic acid and its derivatives.

Fermentation Test. Robert's Differential Density Method. Take the specific gravity of the urine before adding the yeast and record it. Mix well 2 fluid ounces $(60 \mathrm{cc}$.) of urine with $1 / 4$ cake of compressed yeast in a bottle. Set aside for 24 hours in a moderately warm place. After the fermentation filter and take the specific gravity again and subtract from that taken before Each degree of the remainder represents one grain of glucose to the fluid ounce. Multiply by 0.219 to get the percentage. Thus: Specific gravity before fermentation, 1035; specific gravity after fermenta- 

tion, 1015. 1035-1015=20 degrees of density lost, or 20 grains of sugar to the fluid ounce. This test is conclusive as to the presence of sugar, though it is not absolutely accurate as to quantity.

\section{VI.}

Bile in the Urine. In a number of pathologic conditions the elements of the bile are excreted in the urine. The bile pigments, bilirubin and biliverdin, may occur along with the bile salts, sodium glycocholate and taurocholate, or the bile salts alone may be present.

Urine containing the bile pigments is colored a yellow brown or brownish green. It forms an intense yellow froth on agitation. It stains paper or linen a permanent yellow. ${ }^{*}$ The bile pigments are found in jaundice, functional disorders of the liver (acute and chronic biliousness); organic diseases of the liver apart from jaundice (carcinoma, amyloid disease, cirrhosis); diseases of the spleen; fever; hemolytic diseases (anemia, leucocythemia and scurvy).

Gmelin's Test. (Nitric acid containing nitrous acid). $\dagger$ Place a few drops of the suspected urine in a white porcelain dish and near them a few drops of the impure nitric acid; let the fluids run together and the usual play of colors is observed.

Put some urine in a test tube and carefully pour in some of the yellow impure nitric acid, until it forms a stratum at the bottom. If the bile pigments are present at the line of junction of the fluids, a play of colors takes placefrom above downwards - green, blue, violet or dirty red, and yellow. Nearly all urines give a play of colors, but green is the necessary and characteristic color to prove the presence of the bile pigments.

Pettenkofer's Test for Bile Acids. Take about $2 \mathrm{cc}$. of suspected urine in a test tube and add 4 drops of a $10 \%$

*A little omnivorous or carnivorous bile may be added to the normal urine to demonstrate the tests if no icteric urine is available.

$\dagger$ Nitrous acid may be prepared by heating a little nitric acid to which a small amount of starch has been added. 

solution of the cane sugar. Add strong sulphuric acid, drop by drop; cooling the tube in a dish of cold water immediately after adding the acid. Not more than $2 \mathrm{cc}$. of the acid should be used. Too much heat causes carbonization of the sugar and the test is ruined. If bile acids are present, the fluid at first becomes opaque, then clear and successively brown, red and purple. It may require an hour or more to accomplish the test. This reaction depends upon the production of furfurol by the destruction of the sugar when the sulphuric acid is used. Furfurol in turn combines with cholalic acid, formed by the action of the sulphuric acid on the bile acids, giving the color.

Pettenkofer's test may also be quite satisfactorily performed more quickly by putting a little of the suspected urine in a porcelain capsule, adding a few drops of a solution of cane sugar and then a few drops of strong sulphuric acid, keeping the mixture cool to prevent carbonization.

Udranszky has modified Pettenkofer's test by using furfurol directly instead of waiting for its formation by the action of the sulphuric acid upon the sugar. His procedure is as follows: One cubic centimeter of the urine is treated with one drop of a $0.1 \%$ solution of furfurol and slowly superposed upon $1 \mathrm{cc}$. of sulphuric acid, care being taken to prevent too great heating of the mixture. A purple color appears at the plane of contact, that gradually extends upward into the superposed solution; on standing, the color turns bluish. In alcoholic solution a green fluorescence is seen. The pigment gives a typical spectrum.

Hay's Test. Use two test tubes; in one place a little normal urine and in the other some of the suspected urine. Leave the test tubes in the rack in order to prevent agitation. Drop a little finely powdered sulphur upon the surface of the urines. If bile or biliary acids are present in the suspected urine the sulphur will sink at once to the bottom of the tube, while in the normal urine it will remain upon the surface or but a slight amount may sink, if the tubes are not agitated.

Chloroform as a test for bile is quite satisfactory. Agitate a few drops of chloroform with the suspected urine in a test tube. If bile be present the chloroform becomes turbid 

and acquires a yellowish hue, the depth of which is in proportion to the amount of bile present.

To some of the suspected urine add a little bromine water $(1 \%)$. If bile is present a green ring should appear.

Blood. Blood is sometimes a constituent of the urine in disease. It may occur in two forms: 1. As hematuria, when the blood coloring matter is present in the urine in combination with blood corpuscles. 2. As hemoglobinuria, when no blood corpuscles are present and the blood pigment or hemoglobin is in solution in the urine.

Hematuria may have its source (1) in the kidneys due to injuries; acute nephritis; acute acerbation of chronic nephritis; diseases of renal vessels (embolism, thrombosis, aneurism, stasis); amyloid kidney (very rarely); infective fevers (smallpox, scarlatina, typhoid fever, etc.); certain blood diseases (scurvy, purpura, hemophilia); parasitic diseases (echinococcus). 2. The renal pelvis and ureters due to renal calculi; tuberculosis; rupture of neighboring abscesses; parasites. 3. The bladder due to calculi; cancer and other tumors; diphtheric cystitis; varicose veins; injuries. 4. The urethra due to injury (catheterization, impaction of calculi, etc.). 5. Extraneous discharges as the menstrual flow, etc.

Hemoglobinuria has been observed in severe infectious diseases (typhoid fever, scarlatina, etc.); in conditions of blood dissolution (scurvy, purpura, etc.); in skin burns, sunstroke, etc.

Heller's Blood Test is made by adding a little caustic soda solution to some urine in a test tube and heating. The precipitated phosphates are colored reddish brown and fall in a thick cloud to the bottom of the tube.

Almen's Test. Add a few drops of freshly made 5\% alcoholic tincture of guaiacum to the suspected urine. Shake well and add a few drops of hydrogen dioxide or of old oil of turpentine, and the mixture will turn greenish blue. The hemoglobin will change the color of the precipitate to blue. The test will not respond to a small quantity of blood.

Hemin Test. If some blood or sediment supposed to contain blood be heated gently with some glacial acetic acid and a trace of sodium chloride, under a cover glass and then slowly evaporated in the air, brownish yellow rhombic crystals of hemin are found. The slide should be heated 

two or three times, running a drop of the acid under the cover each time.

The spectroscope and microscope are also used in blood tests.

Melanine. In certain cases of melanosis this pigment appears in the urine, which, when emitted is clear, but gradually becomes of a deep brown, or even black color.

Ordinarily melanine exists in solution in the urine, but sometimes in the form of brownish or black sediment, recognizable by microscopic examination. Melanine may possess a diagnostic significance when the melanosis is beyond the reach of examination by eye or touch.

It may disappear from the urine when the disease is arrested, or it may remain stationary. Oxidizing agents, such as chromic acid and fuming nitric acid, transform the principle melanine, causing gradually with the first and immediately with the second a black coloration. According to Zeller, the most delicate test for melanine is bromine water. With melanine it gives at first a yellow precipitate, which gradually blackens. Urobilin gives a yellow precipitate with the same reagent, but it does not blacken.

The practical significance of this condition (melanuria) is greatly limited by the fact that the urine may contain a large quantity of melanine in wasting diseases, whilst that derived from individuals suffering from melanotic cancer or sarcoma may be entirely free from it. Senator has recently confirmed this view by a series of clinical observations. Nevertheless, as an adjunct in diagnosis, the tests given are of undoubted utility. 



\section{VII.}

\section{QUANTITATIVE ANALYSIS.}

Centrifugal Method. The centrifuge as ordinarily used gives the bulk percentage of the constituents, this percentage being arrived at arbitrarily as a result of numerous determinations upon normal urine. Knowing the normal bulk percentage it is not difficult to determine an abnormal or pathologic amount of a substance as indicated by its excess or deficiency, and the method is therefore a convenient and expeditious one for clinical purposes. To a beginner, however, the method as commonly employed, is misleading in that the bulk percentage and the irue or actual percentages are widely different. Take for example the phosphates of the urine. The normal bulk percentage as given by the centrifuge is $8 \%$ for man; $1 \%$ or less for the horse. Whereas, the real amount of phosphates present as $\mathrm{P}_{2} \mathrm{O}_{5}$ in the urine of man for the whole 24 hours ( $1500 \mathrm{cc}$. of urine) is only about 3.5 grams, or in $1000 \mathrm{cc}$. about 2.5 grams, or a true percentage of $0.25 \%$. The true percentage may be calculated

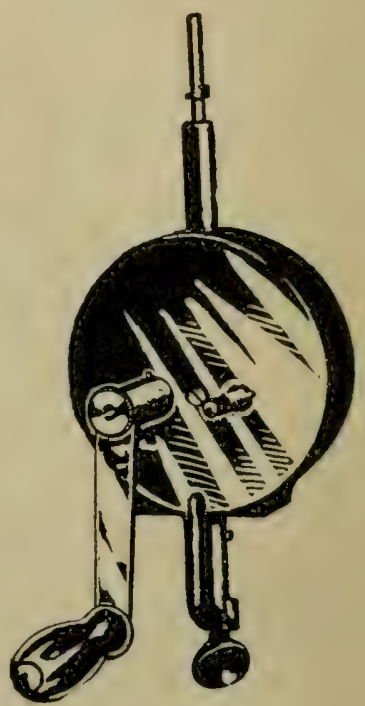

FIG.9

Hand Centrifuge. from the centrifuge by giving to each $0.1 \mathrm{cc}$. of precipitate a determined value with reference to the amount of $\mathrm{P}_{2} \mathrm{O}_{5}$ present.

Each centrifuge tube is graduated to $15 \mathrm{cc}$. The first $10 \mathrm{cc}$. are divided so that each cubic centimeter is divided into 10 parts, each part representing $0.1 \mathrm{cc}$. The remaining $5 \mathrm{cc}$. are for holding the test reagents which are added to the $10 \mathrm{cc}$. of urine. The first cubic centimeter because of the tapering end, will admit of finer graduation than the remainder of the tube. The first half of the cubic centimeter is divided into $1 / 40 \mathrm{cc}$. (or $.025 \mathrm{cc}$.) in order that small amounts of precipitate may be accurately read; the second half of the cubic centimeter is divided into $1 / 20 \mathrm{cc}$. (or $.05 \mathrm{cc}$.); while the remaining $9 \mathrm{cc}$. are each divided into $1 / 10 \mathrm{cc}$. (or $0.1 \mathrm{cc}$.) as above stated. It is convenient to take the $1 / 10 \mathrm{cc}$. 



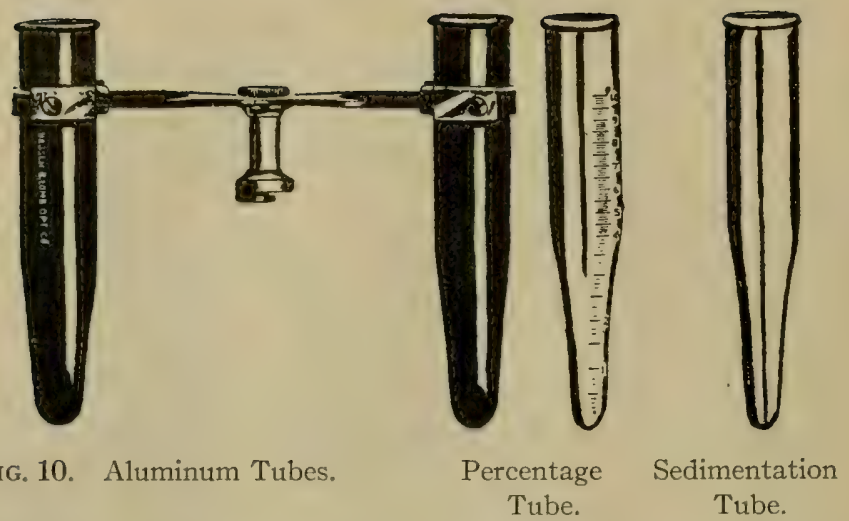

as the unit in the calculation of the true percentage. By taking the average of a great number of control tests with the burette it has been found that $1 / 10 \mathrm{cc}$. of the precipitate, in the case of the phosphates, represents 0.13 gram of $\mathrm{P}_{2} \mathrm{O}_{5}$ in 1000 cc. of urine.

If albumin is present, remove it by adding a little acetic acid and applying heat. Filter and test the filtrate for the inorganic constituents.

With the horse urine care must be exercised in adding the reagents. The large amount of carbonates present cause considerable effervescence, from the liberation of the $\mathrm{CO}_{2}$, and some of the urine is likely to flow over the tube. Add a little of the reagent and when the effervescence has ceased add a little more until the required amount has been used.

Phosphates. Fill the graduated glass tube to the $10 \mathrm{cc}$. mark with the urine to be tested. Add 1 cc. of glacial acetic acid and $4 \mathrm{cc}$. of $5 \%$ uranium nitrate solution to reach the $15 \mathrm{cc}$. mark. Invert the tube several times and revolve in the centrifuge for three minutes. If, after revolving three minutes at 1000 revolutions per minute, the precipitate comes up to the eighth $0.1 \mathrm{cc}$. line of the tube $(0.8 \mathrm{cc}$.) multiply 0.13 by 8 for the product, which is 1.04 grams of $\mathrm{P}_{2} \mathrm{O}_{5}$ in $1000 \mathrm{cc}$. If the total amount of urine for the 24 hours is $1400 \mathrm{cc}$., the amount of $\mathrm{P}_{2} \mathrm{O}_{5}$ present in this quantity can easily be calculated by the following proportion: 1.04 gm. $\mathrm{P}_{2} \mathrm{O}_{5}: 1000 \mathrm{cc}$. : : X : 1400. In which the value of $\mathrm{X}$ is found to be 1.456 grams of $\mathrm{P}_{2} \mathrm{O}_{5}$ in 24 hours. Make the same determination with the urine of the horse. 

Chlorides. Fill the graduated tube to the $10 \mathrm{cc}$. mark with the urine. Add 15 drops or $1 \mathrm{cc}$ of nitric acid to prevent precipitation of the phosphates. Then fill to the $15 \mathrm{cc}$. mark with the silver nitrate solution (1 to 8 ). Invert the tube several times to thoroughly mix the reagents. Revolve in the centrifuge for intervals of three minutes until the precipitate no longer settles. The average amount of precipitate for man is from $0.5 \mathrm{cc}$. to $0.8 \mathrm{cc}$; for the horse about $0.5 \mathrm{cc}$. The value of each $0.1 \mathrm{cc}$. is 1.3 of a gram of $\mathrm{NaCl}$ per 1000.

Sulphates. These are estimated as insoluble salts of barium. Fill the graduated tube to $10 \mathrm{cc}$. mark with fresh urine and add $5 \mathrm{cc}$. barium chloride solution, (4 parts barium chloride, 1 part hydrochloric acid, 16 parts distilled water).

The amount of precipitate for man and horse is about $.075 \mathrm{cc}$. normally. The value of each 0.1 precipitate is 2.4 grams of $\mathrm{SO}_{3}$.

In the above tests write down in your notes the amount of each constituent for the 24 hours considering $1250 \mathrm{cc}$. as the total amount of urine passed.

Quantitative Estimation of Uric Acid. This estimation is difficult and time-consuming and is generally regarded as inexpedient for clinical purposes. The following methods are described because they are more suitable for clinical work although less accurate than the more elaborate methods of Salkowski, Ludwig, Hopkins and others.

Ruhemann's Uricometer for the rapid estimation of uric acid consists of a graduated tube with a glass stopper. It is a colorimetric method, in which iodine with carbon bisulphide serve as indicators.

The following directions accompany each apparatus:

Fill the glass tube to the lowest mark $S$ with carbon bisulphide. (It is not necessary for the tube to be quite dry, but there must be no drop of liquid at the bottom).

The lowest part of the convexity (double meniscus) has to be even with mark $S$, see diagram.

Add a solution consisting of iodine, $0.5 \mathrm{gm}$; potassium iodide, $1.25 \mathrm{gm}$; absolute alcohol, $7.5 \mathrm{gms}$; glycerine, $5 \mathrm{gms}$; distilled water to make $100 \mathrm{gms}$. 



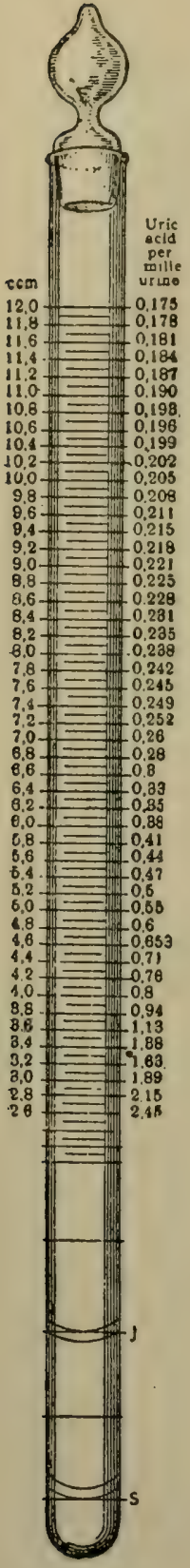

FIG. 11

Ruhemann's Uricometer.

Fill up so that the base of the upper arch of the double meniscus is on a level with mark $J$ as shown in the illustration.

Then add the urine to be examined, which has to be at a temperature of $18^{\circ}$ Centigrade, to the mark of $2.45(2.6 \mathrm{ccm}$.)

Close the tube with glass stopper and shake well when the carbon bisulphide will become a dark copper brown color.

After adding more urine under continued strong shaking, the carbon bisulphide will absorb all free iodine and the mixture will look like urine.

Slowly adding more urine will change the yellow foam, created by the shaking, into white foam.

The color of the carbon bisulphide will turn pink after a while.

Should this color remain the same after the apparatus has been shaken repeatedly and turned upside down, add another drop of urine and keep up the same procedure until only a slightly reddish coloration of the carbon bisulphide remains.

Now shake again vigorously and the carbon bisulphide will turn porcelain white and the urine will look like cloudy whey.

To recapitulate:- The adding of urine has to be stopped as soon as the carbon bisulphide shows only a slightly reddish tint, because this will disappear entirely after repeated shakings. The test is finished when the indicator appears snow-white, a sign that all iodine has been neutralized by the urine.

To get rid of the remaining foam move the tube a few times slowly to a horizontal position, then open the stopper a little, to allow all liquid to settle in the tube.

The proportion of uric acid is then read off the upper scale (per thousand of urine). The percentage is obtained by putting 

a 0 in front. If the upper meniscus line of the urine is between any of the $0.1 \mathrm{ccm}$. marks, the upper number should be read.

The figures to the left of the apparatus refer to the number of cc. of urine added to the mixture.

Should the urine contain less uric acid than the apparatus will in this way indicate, add the iodine solution to the mark half way between $S$ and $J$ and read after each reaction the half values.

The vessel in which the urine is to be kept, must not be cleaned with soda.

If the urine shows an acid reaction, it can be used at once, but if it should be alkaline, it has to be made acid by adding diluted acetic acid. Cloudiness is of no importance.

If the urine contains a considerable sediment of sodium urate it should be well shaken.

Strong colorations of the urine do not affect the action of carbon bisulphide.

Traces of sugar and albumin do not disturb the result.

If there is a very large percentage of albumin or traces of blood or pus, these pathologic substances have to be coagulated by boiling and the urine is filtered.

The apparatus is not satisfactory for determining the uric acid in the urine of the horse.

(The uricometer may be purchased of Eimer \& Amend, New York).

Estimation of Uric Acid by Weight. $20 \mathrm{cc}$. of hydrochloric acid are added to $200 \mathrm{cc}$. of urine and the mixture set aside for 24 hours. Uric acid crystals form and collect on the sides of the vessel. This may be collected on a weighted filter and washed thoroughly with water. The filter and uric acid are dried and weighed until there is no further loss of weight. The weight of the filter paper is deducted and the result gives the amount of uric acid in 200 c.c of urine, from which the amount in 24 hours can be calculated.

Urea. Doremus Ureometer Test. Fill the long arm and bend of the ureometer with the hypobromite solution. (Hypobromite of sodium is prepared by mixing $2 \mathrm{cc}$. of bromine with . $23 \mathrm{cc}$. of a solution of caustic soda, 40 grams to $100 \mathrm{cc}$. of distilled water. To this mixture add an equal volume of distilled water). With a thoroughly washed pipette draw up exactly $1 \mathrm{cc}$. of urine and pass the pipette through the bulb of the ureometer as far as it will go in the bend. Compress the bulb of the pipette gently and steadily. The urine will rise through the hypobromite, and the 

urea instantly decompose, giving off nitrogen gas. Withdraw the pipette after the urine has been expelled, taking care not to press the bulb hard enough to drive the air out with the urine, and read the volume of gas, after allowing the froth to subside.

Each division mark on the ureometer indicates 0.001 gram of urea in $1 \mathrm{cc}$. of urine. The quantity of urea voided in 24 hours is ascertained by multiplying the result of the test by the number of cc. of urine passed during that period.

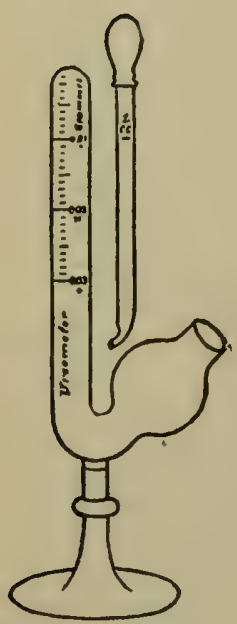

FIG. 12

Doremus

Ureometer.

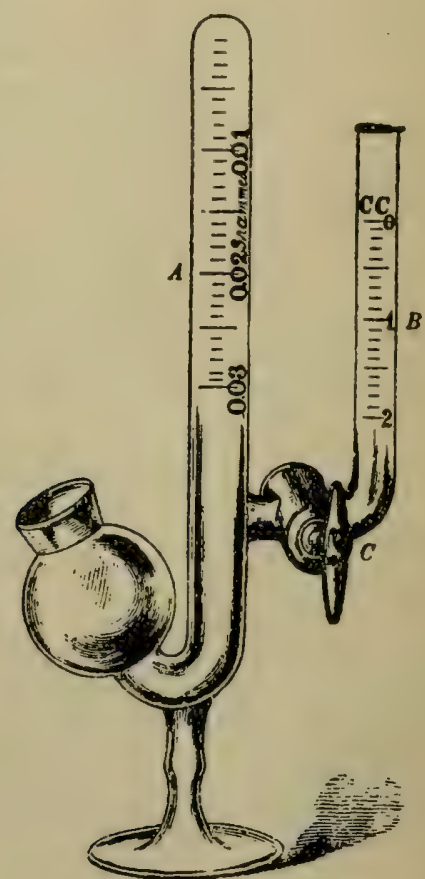

FIG. 13. Hind's modification of Doremus Ureometer.

The $\mathrm{CO}_{2}$ resulting from the decomposition of the urea is absorbed by the excess of soda in the hypobromite solution, and nitrogen is evolved. $37.1 \mathrm{cc}$. of moist nitrogen gas measured under the ordinary conditions of experiment may be taken to represent 0.1 gram of urea. 1 gram of urea corresponds to 13.72 grams of muscular tissue. 

Albumin. Esbach's Method. Fill the tube with the suspected urine to the letter $\mathrm{U}$, then add the reagent to the letter $\mathrm{R}$. (The reagent is made by mixing 10 grams of picric acid and 20 grams of citric acid and adding enough water to make 1 liter. It is said that acetic acid may be substituted for the citric with as good results). Invert the tube a number of times so that the contents may be thoroughly mixed. Close the tube tightly with the rubber stopper and set aside for 24 hours, after which the amount of dried albumin in one liter of urine can be read in grams on the tube. The percentage is obtained by dividing by ten. Thus, if the coagulum stands at 3 , the urine contains three parts of albumin per thousand, or $0.3 \%$. If the albumin is very abundant (above four) the urine should be diluted to obtain an accurate result. Less than 0.5 per thousand of albumin cannot be accurately estimated by this method.

Caution. The coagulable substance found in

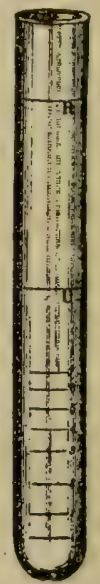

FIG. 14

Esbach's Albuminometer the urine of Bright's disease is a mixture of "serum-albumin" and "serum-globulin" or "para-globulin." Saturation of the urine with crystallized magnesium sulphate precipitates the serum-globulin, leaving the serum-albumin in solution.

Centrifuge Test. Fill the graduated tube with urine up to the $10 \mathrm{cc}$. mark; add $5 \mathrm{cc}$. of Esbach's reagent. Invert the tube several times in order to thoroughly mix the fluids. Place the tube in the centrifuge and revolve for three minutes. Read off the amount of the precipitate; each $0.1 \mathrm{cc}$. is the equivalent of 0.21 of a gram of dry albumin per $1000 \mathrm{cc}$. of urine.

Ferrocyanide Test with Centrifuge. The percentage tube is filled to the $10 \mathrm{cc}$. mark with the urine to be tested. Add $1 \mathrm{cc}$. of acetic acid and $4 \mathrm{cc}$. of a $5 \%$ solution of potassium ferrocyanide. Proceed as in the preceding test. Each 0.1 cc. of precipitate is the equivalent of 0.21 gram of dry albumin per $1000 \mathrm{cc}$. of urine.

Sugar. Einhorn's Fermentation Saccharometer. 

Determine the specific gravity. Add $10 \mathrm{cc}$. of the suspected urine to $90 \mathrm{cc}$. of distilled water Put in a flask, add about one gram of compressed yeast and agitate

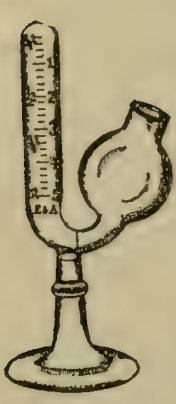

FIG. 15

Einhorn's

Saccharometer. thoroughly. Pour $10 \mathrm{cc}$. of the mixture into the bulb of the saccharometer and, by inclining the apparatus, the fluid will displace the air in the cylinder and remain there by atmospheric pressure. $\mathrm{Be}$ sure that no air bubbles remain in the cylinder. It is always well to test a normal urine at the same time and in the same way as a control. The mixture of normal urine with yeast will, on the following day, have only a small bubble on the top of the cylinder. If, in the suspected urine, there is also present at the top of the cylinder a small bubble, no sugar is present; but if there is a much larger volume of gas

$\left(\mathrm{CO}_{2}\right)$ it is certain that the urine contains sugar. The apparatus should remain in a moderately warm place. As a result of 'fermentation the sugar is broken up into alcohol and $\mathrm{CO}_{2}$. The changed level of the fluid in the cylinder shows that the reaction has taken place and indicated by the numbers the approximate quantity of sugar present. The scale on the tube is empirical and indicates directly the percentage in the urine.

Shieb's Test for Sugar in the Urine.

Solution No. 1.

Ammonium Sulphate (purest)

1.2 grams.

Copper Sulphate (purest)

2.6 grams.

Distilled Water

50. cc.

Solution No. 2.

Caustic Potash C. P. - $\quad$ - 20 grams.

Distilled Water - - $\quad$ - $50 \mathrm{cc}$.

Dissolve and when, cool, add

Glycerine - $\quad$ - $\quad$ - $50 \mathrm{cc}$.

Ammonia water $0.960 \mathrm{sp} . \mathrm{gr}$. $\quad 300 \mathrm{cc}$.

Add No. 1 to No. 2 and dilute the whole to $500 \mathrm{cc}$. with distilled water. Stopper securely and shake till thoroughly mixed.

Heat one dram of this solution in a test tube to boiling. Add the urine drop by drop, at slow intervals, boil- 

ing after each addition until the blue color has been discharged and the fluid has a light amber color or is colorless.

If the solution is decolorized by 3 minims of urine it contains 9 to 10 grains of sugar per oz.

If the solution is decolorized by 4 minims of urine it contains 7 to 8 grains of sugar per oz.

If the solution is decolorized by 5 minims of urine it contains 5 to 6 grains of sugar per oz.

If the solution is decolorized by 6 minims of urine it contains 4 grains of sugar per oz.

If the solution is decolorized by 7 minims of urine it contains 3 grains of sugar per oz.

If the solution is decolorized by 9 minims of urine it contains 2 grains of sugar per oz.

If the solution is decolorized by 10 to 17 minims of urine it contains 1 grain of sugar per oz.

If the urine contains more than 10 grains of sugar to the ounce it must be diluted with an equal quantity of water, and the number of grains per ounce multiplied by two.

A very similar preparation, accurately compounded, is on the market under the name of Whitney's Reagent. It is for sale by the Norwood Chemical Co., 105 W. 40th St., New York.

The following table gives the amounts of sugar in analytical testing with Whitney's Reagent:

If reduced by
1 minim
2 minims
3
4

It contains to the oz. Percentage. 16 grains or more

3.33

8 grains

1.67

5.33 grains

1.11 .

4

0.83

3.20

0.67

2.67

0.56

2.29

0.48

2

0.42

1.78

0.37

1.60

0.33

(1

0.21 ) 



\section{BENEDICT'S QUANTITATIVE METHODS}

Copper Sulphate crystals -

18 grams

Sodium Carbonate $\quad$ - $\quad$ - 200

Sodium Citrate $\quad-\quad \ldots \quad-\quad-\quad 200$

Potassium thiocyanate - - $\quad$ - 125 “

Potassium ferrocyanide ( $5 \%$ Sol.)

$5 \mathrm{cc}$.

Distilled water to - $\quad$ - $1000 \mathrm{cc}$

Preparation: Dissolve the sodium citrate, sodium carbonate and potassium thiocyanate in $800 \mathrm{cc}$. of distilled water with the aid of heat and filter. Dissolve the copper sulphate separately in $100 \mathrm{cc}$. of distilled water and mix the two solutions slowly with constant stirring; then add the potassium ferrocyanide solution. Cool and dilute with distilled water to exactly $1000 \mathrm{cc}$.

Application. To $25 \mathrm{cc}$. of Benedict's solution in a small beaker add from 4 grams to 5 grams of anhydrous sodium carbonate and heat the mixture to boiling over a wire gauze until the carbonate has been brought into solution. Add a little distilled water occasionally to make up for evaporation.

Place the urine under examination in a burette and run it into the hot Benedict Solution rather rapidly until the formation of a heavy chalk-white precipitate is noted and the blue color of the solution lessens perceptibly in its intensity. From this point in the determination from 2 to 10 drops of the urine should be run rather slowly into the boiling Benedict Solution at one time, boiling the solution vigorously for about 15 seconds after each addition. Complete reduction of the copper is indicated here as in Fehling's original method, by the complete disappearance of all blue color and the white precipitate of cuprous thiocyanate. The end-point here, however, is very sharply defined, contrary to the conditions in the older method.

To preverit the annoying bumping which often interferes with the titration, a medium-sized piece of washed absorbent cotton may be introduced into the solution. This cotton may be stirred about through the solution as the titration proceeds and the bumping thus eliminated. Calculation. Twenty-five cubic centimeters of Bene- 

dict's Solution is completely reduced by 0.05 gram of dextrose. If $Y$ represents the number of cubic centimeters of urine necessary to reduce the $25 \mathrm{cc}$. of the solution we have the following proportion $Y: 0.05:: 100: X$ (percentage of . dextrose).

Ehrlich's Diazo-reaction. This test has been recommended for the diagnosis of typhoid fever in man. It is stated that the reaction will also occur in some other diseases, but in spite of this fact the test is of value as an aid in the diagnosis. Two solutions are prepared as follows:

1. Sulphanilic acid Hydrochloric acid 2 gms.

Distilled water

$50 \mathrm{cc}$.

$1000 \mathrm{cc}$.

2. A. $0.5 \%$ solution of sodium nitrate.

In performing the test, 50 parts of No. 1 and 1 part of No. 2 are mixed, and equal parts of this mixture and of the urine in a test tube are rendered strongly alkaline with ammonia. If the reaction be positive, the solution assumes a carmine-red color, which on shaking must also appear in the foam. Upon standing 24 hours a greenish precipitate is formed. The test must not be considered positive unless a distinct coloration extends to and includes the foam on shaking.

\section{VIII. \\ VOLUMETRIC METHODS.}

Chlorides. Mohr's method. Principle: If silver nitrate added to a solution containing sodium chloride, neutral potassium chromate, and an alkaline phosphate, the chloride is first precipitated, then the chromate, and lastly, the phosphate. The formation of the red silver chromate indicates the complete precipitation of the chloride:

Solutions required:

1. Standard solution of silver nitrate:

Fused with silver nitrate 29.075 grams.

Distilled water to make $1000 \mathrm{cc}$. 

2. Saturated solution neutral potassium chromate:

Neutral potassium chromate 10 grams.

Distilled water to make $100 \mathrm{cc}$.

Process. The urine should not be high colored, and should be free from albumin or excess of uric acid or mucus. Dilute $10 \mathrm{cc}$. of the urine with $100 \mathrm{cc}$. of distilled water. Fill a burette with the silver solution to the zero mark. Drop it slowly into the urine, stir it well and occasionally carry a drop of the mixture so as to come in contact with a little of the chromate solution in an evaporating dish. Test in this way until the first trace of orange color appears in the chromate solution. Make sure that the precipitation of the chlorides is complete by adding another drop of the silver solution from the burette. Read off the amount of silver solution used and calculate the result.

Example:

Quantity of urine in 24 hours, 1250 cc.

Silver solution used, - - $7.5 \mathrm{cc}$.

1. cc. silver solution equals .01 gram $\mathrm{NaCl}$

$$
\frac{.01 \times 7.5}{10} \times 1250=9.375 \text { grams. }
$$

Make two or three determinations and take the average for your final result. Do the same in the phosphate and sulphate tests.

If the urine of the horse is very dark colored, it may be filtered through animal charcoal to make it light colored. Some of the chlorides may be held by the charcoal and thus diminish the amount in the urine tested; or the urine may be diluted with an equal volume of distilled water and the result multiplied by two.

\section{Gravimetric Method.}

A more accurate method is the following: If the urine is high colored, and contains albumin, or excess of uric acid and mucus, they must be removed. To do this, measure $10 \mathrm{cc}$. of the urine into a platinum capsule, add 2 grams of pure potassium nitrate, evaporate to dryness, and ignite at a dull red heat to destroy organic matter. When cool, treat the residue with hot water and filter. Acidulate the filtrate with dilute nitric acid, neutralize with carbonate of lime and proceed as above.

Estimation of Phosphoric Acid. (Estimated as $\mathrm{P}_{2} \mathrm{O}_{5}$ ). By uranium acetate or nitrate. This method is based upon the fact 

that when a solution of acetate or nitrate of uranium is added to a solution containing soluble phosphates, sodic acetate and free acetic acid, all the phosphoric acid will be precipitated as phosphate of uranium. This precipitate is of light yellow color, insoluble in acetic, but soluble in hydrochloric acid. The point of completion of the phosphoric reaction may be ascertained by placing with a glass rod a drop of the yellow mixture in contact with a drop of potassium ferrocyanide solution upon a white plate or filter paper. As soon as there is the slightest excess of the uranium solution after the phosphates have been satisfied, a brown precipitate will result in the mixture due to formation of ferrocyanide of uranium. The cochineal solution, noted below, serves as a better indicator for the horse urine than the ferrocyanide. The following solutions are used:

1. A Solution of Cochineal prepared by boiling 40 grams of cochineal in $800 \mathrm{cc}$. of water. When cool add $200 \mathrm{cc}$. of alcohol and filter.

Or a solution of potassium ferrocyanide.

Potassium ferrocyanide,

Distilled water

$100 \mathrm{cc}$.

5 grams.

2. Solution of Sodium Acetate.

Sodium acetate,

100 grams.

Acetic acid,

Distilled water to make

- $100 \mathrm{cc}$.

$1000 \mathrm{cc}$.

The following procedure is of use in standardizing the uranium solution.

3. Standard Solution of Disodic Hydric Phosphate, made by dissolving 10.0845 grams of the crystallized salt in water and diluting to 1 liter. Each cc. of this solution contains .002 of a gram of $\mathrm{P}_{2} \mathrm{O}_{5}$. In 5 cc. there is 0.1 gram of $\mathrm{P}_{2} \mathrm{O}_{5}$.

4. Uranium Acetate. Since this cannot be obtained sufficiently pure to be weighed out and used directly we make a solution of it of indefinite strength and standardize it with other solutions.

It has been found best to make the solution of uranic acetate of such a strength that each cc. will precipitate .005 of a gram of $\mathrm{P}_{2} \mathrm{O}_{5}$. Consequently every $2 \mathrm{cc}$. of the uranium acetate should be made equal to every $5 \mathrm{cc}$. of the sodic phosphate, or upon adding $20 \mathrm{cc}$. of the uranium acetate to $50 \mathrm{cc}$. of the sodium phosphate and then touching the plate or paper which has been moistened with the cochineal solution with the drop of the mixture, we should just get the green color.

Put $50 \mathrm{cc}$. of the sodium phosphate with $5 \mathrm{cc}$. of the sodium acetate solution into a beaker. To this add slowly from the burette, the uranium acetate, testing occasionally, for the color on the paper. Suppose that on the addition of $8 \mathrm{cc}$. from the burette, the color is obtained, then $8 \mathrm{cc}$. of the uranium acetate are as strong as 20 should be, and for every $8 \mathrm{cc}$. of the uranium solution that we have, $12 \mathrm{cc}$. of water should be added. If it should 

require more than $20 \mathrm{cc}$. to produce the color, the uranium solution must be concentrated by evaporation or more of the solid salt added. The solution has now been graduated or standardized.

Application. $50 \mathrm{cc}$. of the clear urine, with $5 \mathrm{cc}$. of the sodium acetate solution are poured into a beaker and heated; to this the uranium acetate is slowly added from the burette. The mixture is constantly stirred with a glass rod, which should be applied frequently to the cochineal solution. As soon as the green color is obtained the process is completed. Read from the burette the amount of uranium acetate solution used.

Example. Urine in 24 hours equals $1180 \mathrm{cc}$. Solution uranium acetate used equals $24.3 \mathrm{cc}$.

$$
\frac{.005 \times 24.3 \mathrm{cc} .}{50} \times 1180=2.86 \text { grams } \mathrm{P}_{2} \mathrm{O}_{5} \text {. }
$$

A shorter and approximately accurate method based on the above is as follows: To $10 \mathrm{cc}$. of the urine in a test tube add $1 \mathrm{cc}$. of sodium acetate and $1 \mathrm{cc}$. of the cochineal solution and boil. Add $5 \%$ uranium nitrate solution, 1 minim at a time, boiling after each addition, until the mixture turns green. Each minim of the uranium solution represents 0.046 gram of $\mathrm{P}_{2} \mathrm{O}_{5}$ in $1000 \mathrm{cc}$. of urine. Multiply .046 by the number of minims required to produce the green color, and the product will represent the amount in grams of $\mathrm{P}_{2} \mathrm{O}_{5}$ per liter in the given sample of urine.

Estimation of Total Sulphuric Acid. (Estimated at $\mathrm{SO}_{3}$ ). Dissolve 30.5 grams of pure crystallized chloride of barium in some distilled water and dilute to 1 liter. Each cc. of this solution will equal .01 gram of $\mathrm{SO}_{3}$. A dilute solution of sodium or magnesium sulphate will also be required.

Application. $50 \mathrm{cc}$. of clear urine are poured into a beaker, acidified quite strongly with hydrochloric acid, and heated over the flame. Let the solution boil for about ten minutes, the lamp is then removed and the barium chloride is allowed to flow slowly from the burette into the beaker and it must continue to flow as long as the precipitate is seen to increase. The precipitate is allowed to subside, then more of the barium chloride is added, and this process repeated, until no further precipitate is pro- 

duced. Much time and labor will be saved by filtering a few drops of the solution now and then, and allowing these to drop into the test tube containing some of the dilute sodium or magnesium sulphate. As soon as an excess of the barium chloride has been added, a precipitate will appear in the test tube. Read from the burette the amount of barium chloride used; each cc. of which will indicate .01 of a gram of $\mathrm{SO}_{3}$ in each cc. of urine, and from this the total amount may be calculated.

Example: Urine 2000 cc. Amount of barium chloride solution $25 \mathrm{cc}$.

$$
\frac{.01 \times 25}{50} \times 2000=10 \text { grams } \mathrm{SO}_{3} .
$$

Relation of Urinary Constituents in Normal Human Urine. There is normally a direct proportion within narrow limits, between the solid substances of the urine, which it is desirable to keep in mind.

Relation of Urea to total solids $50 \%$ or one-half.

" " inorganic matter to other solids $30 \%$.

“ " " uric acid to urea $2.5 \%$ or $1 / 40$.

" " " nitrogen in urea to total nitrogen $91 \%$.

" " " phosphoric acid to urea $12.5 \%$ or $1 / 8$.

" " chloride of sodium to urea $40 \%$.

" " the sulphates to total nitrogen $18 \%$

IX.

\section{CHEMICAL EXAMINATION OF URINARY DEPOSITS.}

There is generally a more or less voluminous deposit in the urine after standing for 24 hours; sometimes this deposit is formed in the bladder and sometimes it forms after the emission of the urine; it is important to note this fact. Deposits or sediments should not be confounded with calculi. The former have a pulverunt form while the latter have the appearance of grains or granules of greater or less size.

It is often very easy to determine the nature of the deposit 

by a microscopic examination, but chemical reagents in some cases give more precise results.

With the urine containing sediment, shake thoroughly to distribute it, then pour into a centrifugal tube and revolve until the sediment is completely precipitated. Note the reaction of the urine, whether acid or alkaline. Pour off the clear supernatant fluid. If the urine is acid pursue the following scheme.

Acid Urate of Soda. If the deposit is more or less red, treat with a little boiling water. If the sediment is dissolved it is com-
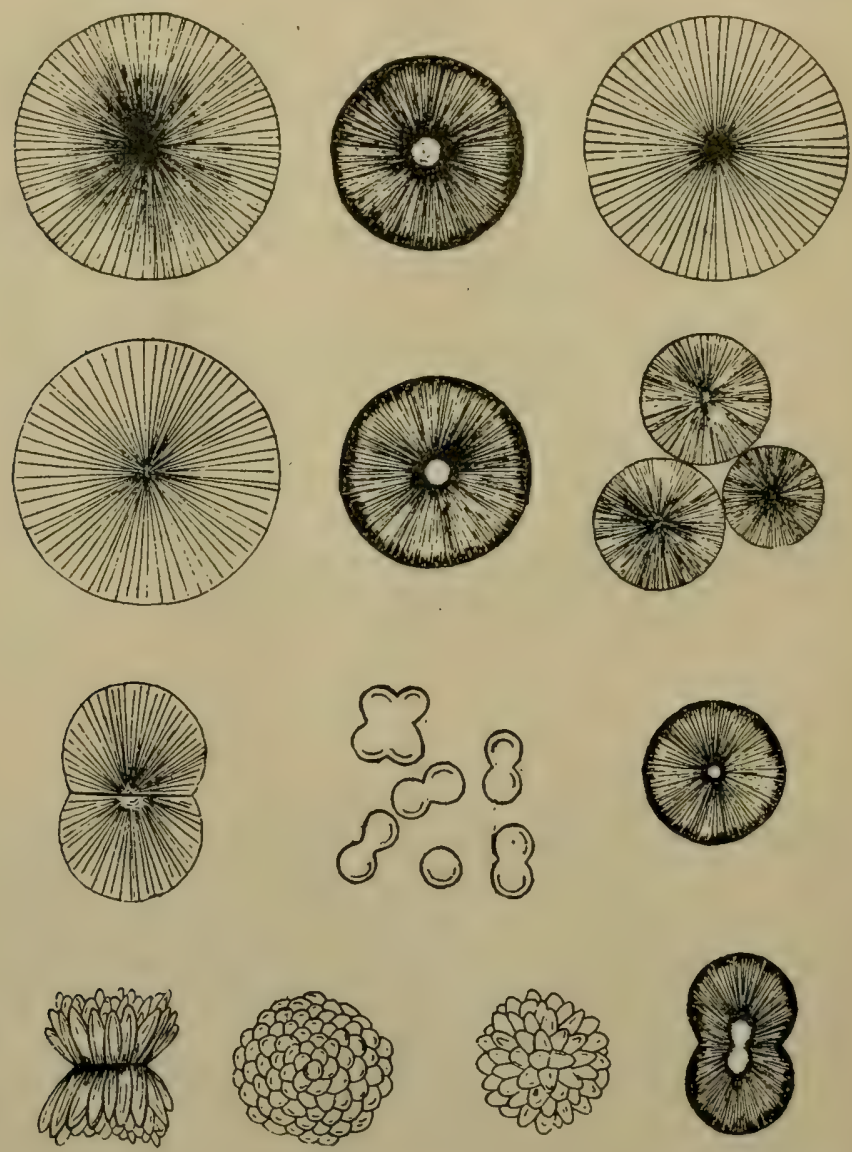

Plate II.

Various forms of Calcium Carbonate Crystals. (Horse Urine) 

posed of the acid urate of soda. Caustic potash does not dissolve it, hydrochloric acid gives a crystalline precipitate, the deposit will give the murexide test. (Page 26).

Uric Acid. If a crystalline deposit, almost insoluble in boiling water, but soluble in caustic potash and giving the murexide test, it is uric acid.

Cystine (very rare). The deposit of crystalline, insoluble in caustic potash but soluble in ammonia and hydrochloric acid.

Pus. A white, dense, mucus deposit, viscid and not often mixing with the urine; caustic potash renders it more viscid; the microscope shows the pus corpuscles. This deposit is rare in acid urines.

Blood. Red deposit; the tincture of guaiac with hydrogen dioxide gives a blue color. The spectroscope gives the characteristic lines in the spectrum. The hemin test will show the characteristic crystals. When blood is present the urine is generally albuminous.

Some deposits not very abundant and having no special chemical reaction may be recognized under the microscope.

\section{Neutral or Alkaline Urine.}

Alkaline Urates. More or less reddish deposits easily soluble in boiling water and giving the murexide test.

Triple Phosphate or Calcium Phosphate. A white deposit,

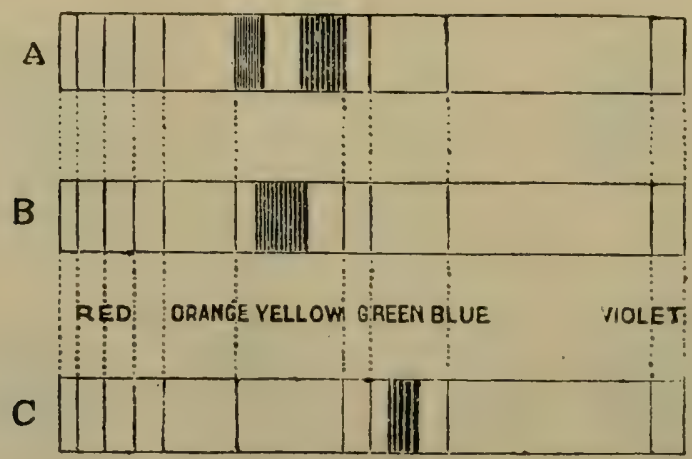

FIG. 16

A.-Spectrum of Oxy-hemoglobin. B.-Spectrum of Reduced Hemoglobin. C.-Spectrum of Urobilin. 

insoluble in boiling water, soluble in acetic acid, does not give the murexide test; acidified with nitric acid, the molybdate of ammonia solution gives a yellowish precipitate in the cold.

Calcium Oxalate. White deposit insoluble in boiling water, also in caustic potash and acetic acid. Soluble in hydrochloric acid or in nitric acid; does not give the murexide test.

Cålc'um Carbona'e. White deposit insoluble in boiling water or in caustic potash. Soluble with effervescence in acetic, nitric or hydrochloric acids, does not give murexide reaction.

Pus, (more frequent in alkaline and albuminous urine). A white, dense, mucus-like deposit, not mixing readily with the rest of the urine. Caustic potash renders it more viscid, the microscope shows pus globules.

Blood. Determined by the guaiacum test, spectroscope, microscope, or hemin test.

From the pathologic point of view the presence of urinary sediments generally indicate an alteration of the secretions.

$\mathrm{X}$.

Microscopical Examination of Urine. If an immediate examination is desired the centrifuge may be used to cause the

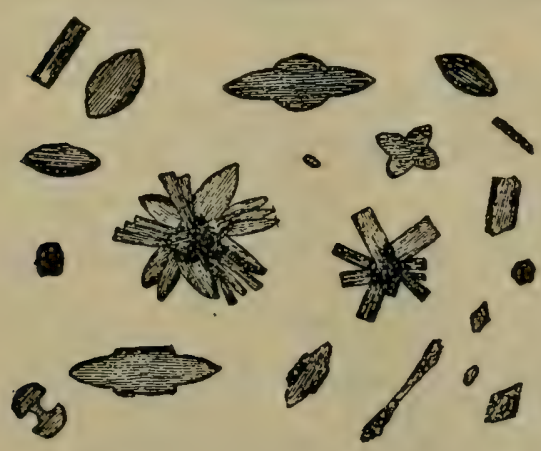

FIG. 17. Uric Acid Crystals. sediment to separate from the urine; otherwise the urine is set aside for some hours in a cool place when the sediment gradually settles to the bottom. The supernatant liquid is poured off and by means of a pipette, camel's hair brush or a wire loop a small portion of the deposit is examined. transferred to a slide and

The deposits may be divided into unorganized and organized sediments. 

Unorganized Sediments. In unorganized sediments two conditions may be considered: 1st, the urine is acid. 2nd, the urine is alkaline.

In acid urine look first for crystals of uric acid diverse in form and reddish brown in color. (Fig. 17). Second, crystals of acid urate of soda, yellowish or red. The crystals are badly formed. Third, crystals of oxalate of lime also found in alkaline urine. (Fig. 18). Fourth, crystals of hippuric acid. Fifth, crystals of calcium sulphate. (4 and 5 are met with rarely in acid urine). Sixth, calcium phosphate. Make sure of the identification by reference to charts.

In alkaline urine look first for ammonio-magnesium phosphates, (Fig. 19), (triple phosphates). Second, bicalcium phosphate crystals. Third, tricalcium or amorphous phosphate crystals. Fourth, crystals of sulphate of lime. Fifth, crystals of oxalate of lime, (also found in acid urine). Sixth,

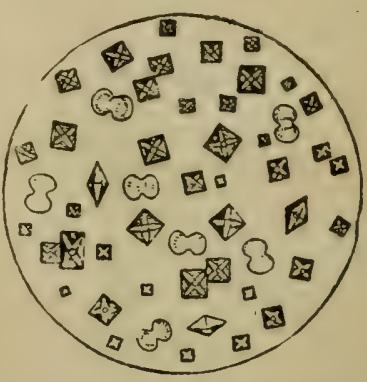

Fig. 18. Calcium Oxalate. crystals of urate of ammonium in the form of yellow colored spheres.

Crystals of cystin, leucin and tyrosin are sometimes encountered in acid or alkaline urines.

ᄂ. Organized Sediments. These are brought more plainly into

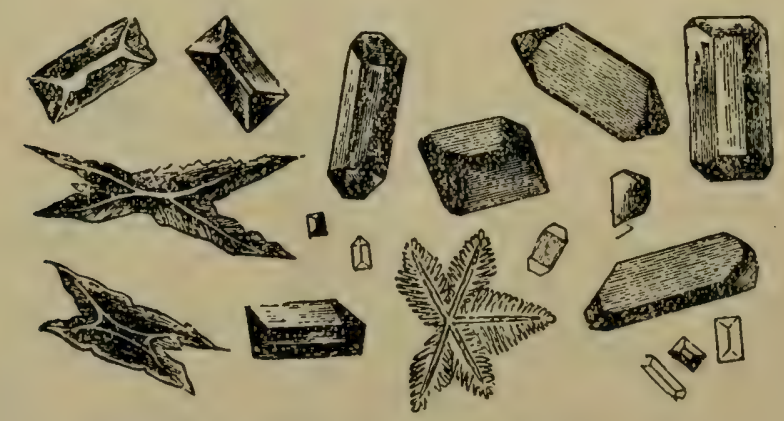

FIG. 19. Triple Phosphates. 

view if the preparations are stained, although this is not universally practised. Organized sediments may be divided into (a) histologic elements, (b) microbic elements.

Of the histologic elements there are frequently encountered, 1st, epithelial cells from the bladder, from the vagina and from the ureter, their presence has no special significance. If, however, they are present in considerable quantity, a lesion of these parts may be suspected. 2nd, cells from the pelvis of the kidney, generally an indication of renal affection. 3rd, cancer cells,

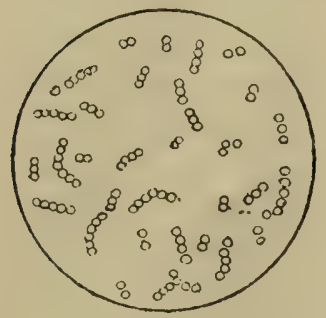

FIG. 20

Micrococcus Ureae. which have a special significance. 4th, hyaline or granular casts, seen more easily in stained preparations; they are encountered frequently in albuminous urine; their presence indicates a mild form of nephritis. 5th, epithelial hemorrhagic or wax casts, of which the hemorrhagic are the most frequent, they are markedly colored and easily recognized on account of the hemoglobin they contain; they contain fine granulations which are not blood corpuscles but possible fragments of them, they indicate a severe form of nephritis. 6th, cylindroid elements drawn out in an irregular and somewhat ribbon-like form; they are the product of the secretion of the urinary epithelium. In the human race they may be found in cases of scarlatina and generally in certain forms of nephritis. In examining for casts it is desirable to examine the urine immediately after emission, as they generally disintegrate rapidly and disappear. 7th, blood corpuscles more or less crenated but easily recognizable by their yellowish tint; the presence of blood corpuscles in the urine indicates a hemorrhage either in the blad-
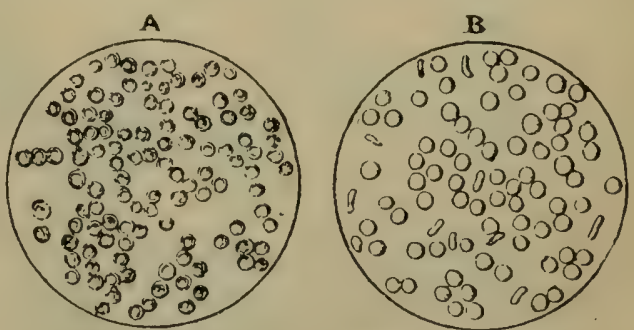

Frg. 21. Red blood corpuscles. A. Normal. der or in the kidney. Sth, pus corpuscles which may have crenated borders, granular contents and appear quite refractive, a drop of 

dilute acetic acid will render the nuclei visible. Pathologically the pus corpuscles indicate a suppuration of some portion of the urinary tract. In the majority of cases it is impossible to say if the pus comes from the bladder or kidney. In cases of cystitis of the neck of the bladder, the last portion of the urine passed contains no pus, while if this trouble be in the kidney this last portion will contain pus. This fact is of some use in diagnosing cystitis of the neck of the bladder. 9th, spermatozoa may be present but are easily recognized by their elongated form. 10th, mucus may be frequently present, but has no very great pat holog i c significance.

Glycogen Cells. If the urinary sediment is stained with dilute L u g o l's Solution, there will be seen, in many specimens of human urine, a number of epithelial cells

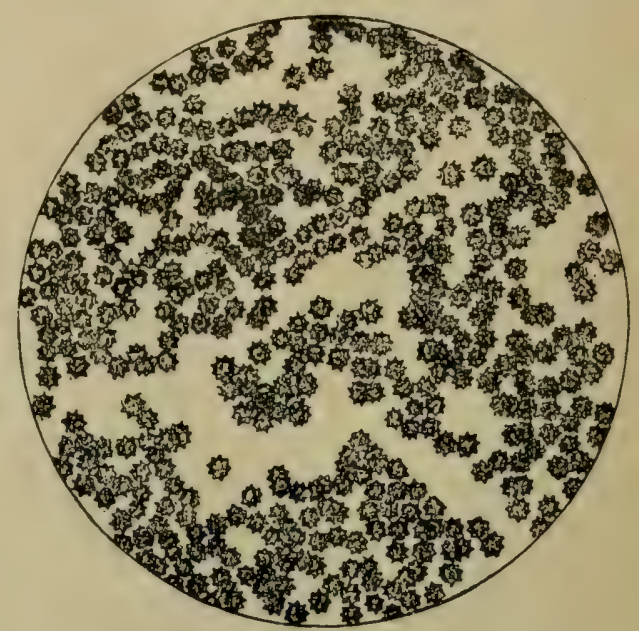

Fig, 22. Red blood corpuscles crenated. present with more or less of their interior stained a deeper brown like glycogen, than in other cells that may be present. As yet, nothing has been determined in regard to their importance.

Amyloid or Amylaceous Bodies. These are small circular or oval bodies which give the starch reaction with an iodine solution. Just what their significance is, is not known. Virchow first described them. They have been found in various tissues and excretions, including the urine normal as well as pathological. In diseases of the kidneys, Veitz and Wederhake found that these amyloids afford us important indications; but that in affections of the bladder they are present in increased quantity. Wederhake therefore suggests that the amyloid bodies have a certain value in differential diagnosis. The absence of the bodies in pathological urine is against catarrhal disease of the bladder; whilst their presence, especially if numerous, in urine which 



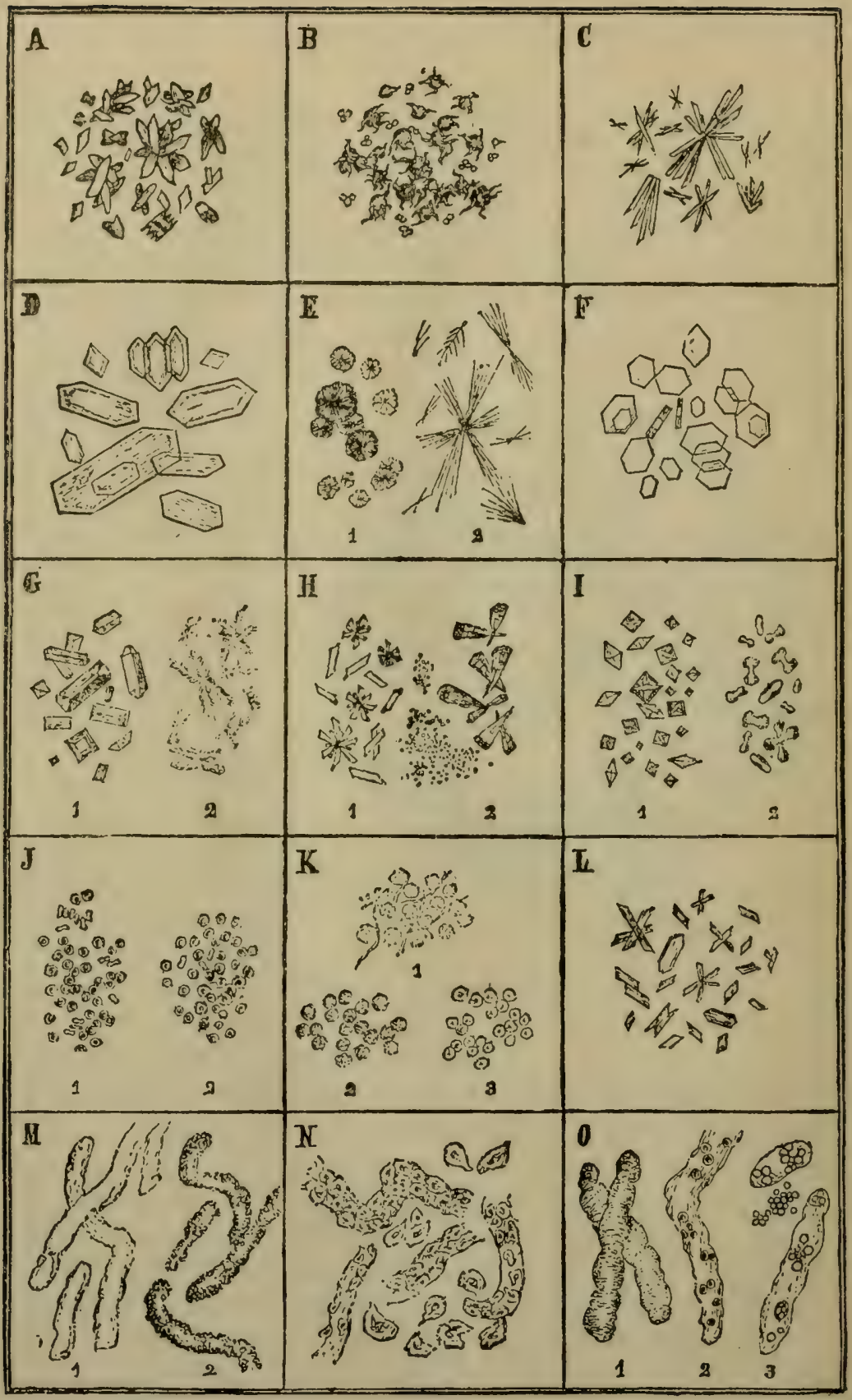

\section{PL.ITE III}

Urinary Sediment. A. Uric Acid. B. Ammonium Urate. C. Acid Sodium Urate. D. Urea Nitrate. E. (1) Leucin and (2) Tyrosin. F. Cystin. G. Ammonio-Magnesium or triple phosphate. H. Calcium Phosphate. I. Calcium Oxalate. J. Blood Corpuscles. K. Pus and Mucus. L. Hemin Crystals. M. (1) Hyaline Casts, (2) Granular Casts. N. Epithelial Casts and Cells. O. (1) Waxy Casts; (2) Casts with Blood Corpuscles; (3) Casts with Fat Globules. (After Simon). 

appears to contain renal elements only, indicates that the bladder is also affected. (Dixon Mann).

The urinary sediment stained with a dilute Lugol's solution show these bodies, when present, varying in color from a blue to a deep blue black color.

Urinary Casts. Although probably observed earlier, Henle is credited in 1842, with first carefully describing the casts moulded in the tubules of the kidneys. Among earlier views, casts were considered as being composed of coagulated fibrin; as products of the secretion of the epithelium of the tubules; as transformed or disintegrated epithelial cells and as products from the blood. A view quite commonly accepted is that casts are the products of the coagulation of albuminous material. 'The fact that the presence of casts in the urine is usually accompanied by the presence of albumin lends force to this view; for the more abundant the albumin, the more likelihood is there of finding casts. From this standpoint, then, casts, may be regarded as albuminous exudates from the blood, with the addition of transformed or destroyed epithelium. In nearly all cases where casts are present, some albumin is found. Occasionally they are sometimes described as being present without the appearance of albumin. This condition is looked upon with some doubt; for it is commonly believed that albumin exists but in

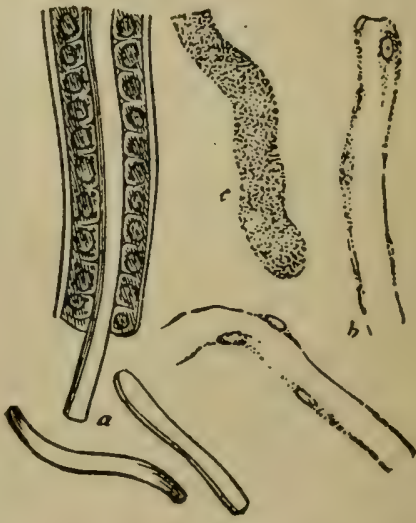

FIG. 23. a. Showing formation of hyaline cast in tubule. b. Hyaline cast with granular deposit. c. Granular cast. an amount too slight to be detected by the ordinary chemical tests.

The presence of casts in the urine is of much diagnostic importance; if found in any quantity, they indicate nephritis particularly if albumin is also present in any amount. It is claimed by some that merely hyperemia of the kidney will cause the appearance of casts in the urine, and that they may sometimes be found when the kidneys are perfectly intact. Mitchell states that two or three hyaline or one or two small granular casts may 

be found in one of every three specimens of the twenty-four hour's urine examined (human). Casts have been described in cases of gastro-intestinal catarrh, in jaundice, in acute and chronic anemia, as well as in nervous affections of different kinds, without accompanying inflammation of the kidneys. There are many, however, who hold that casts are always the products of an inflammatory process, and are, therefore, indicative of renal inflammation. Other evidences or symptoms should also be taken into account.

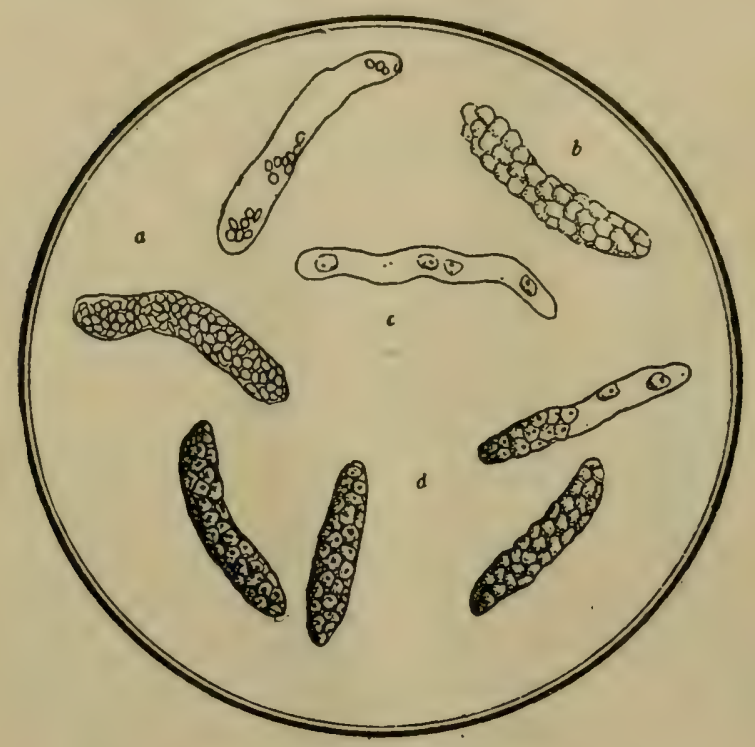

Fig. 24. a. Blood cast and hyaline cast carrying blood cells. b. Pus cast. c. Hyaline cast carrying epithelial cells. d. Epithelial casts. (Greene).
Occasionally it is difficult to find casts even when they are $\mathrm{k}$ now n to exist. Alkaline urine has a tendency to dissolve them. At times they will not settle for hours. Usually if al10 wed to stand six hours, the casts will settle if they are present. The centrifuge will bring them down in a few minutes. A low power of the microscope (150-200 diameters) may be used in the search for casts and will enable the observer to pass over the field quite rapidly. To identify the cast and its structure, a power of 400-500 diameters is required. More than one specimen must always be examined before giving a positive statement as to the presence or absence of casts. False or pseudo-casts have been described; these are believed to be accidental formations, while true casts in general indicate nephritis. 

True casts may appear in three different sizes according to the portion of the tubule in which they are formed. The smaller size originates in the narrow tubule. The next in size comes from the convoluted tubule of the second order (no casts from the convoluted tubule of the first order; i. e., that portion of the tubule nearest the glomerules, appear in the urine, because they are too large to pass through the narrow tubules). The third and largest are those developing in the straight collecting tubules. It is believed a prognostic value may be attached to the size of the casts as well as to their number. Casts from the narrow tubules indicate a mild attack; from the convoluted tubules a severe form, especially in the cortex of the kidney; from the collecting tubules, with the other forms also present, a serious condition of general renal inflammation or unfavorable prognosis. The identity of casts may generally be determined by their uniform width. They are usually longer than they are broad, and have one well-

rounded extremity a nd well defined borders.

True casts are of six varieties; hyaline, epithelial, blood, granular, fatty and waxy casts. In a general wa $y$ the first three varities a re found in an acute form of nephritis. Dur ing the

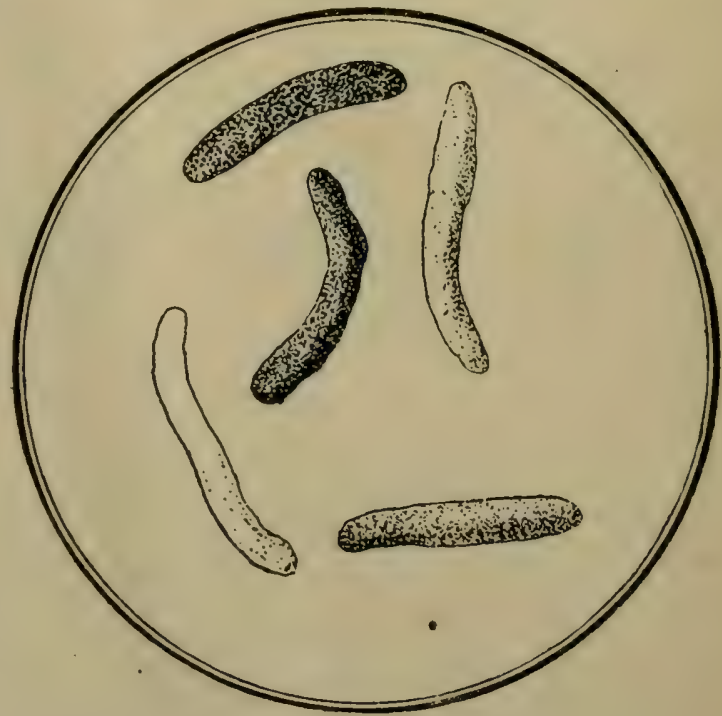

FIG. 25. Granular Casts. (Greene).

first few weeks of the inflammation the last three varieties are not encountered. If the acute condition passes into a subacute, then the granular variety appears, at first in small number, then in larger, with, usually, a considerable number of the hyaline and 

epithelial casts. Fatty and waxy casts are always secondary products, and as a rule not found until a nephritis has existed for some time.

Hyaline casts are colorless, pale, more or less transparent formations soluble in acetic acid. They are of variable size and generally difficult to detect on account of their apparently structureless character. At times a slight granulation may be seen imbedded in or adhering to their matrix and occasionally accidental attachments of pus or fat globules in small numbers.

Epithelial casts

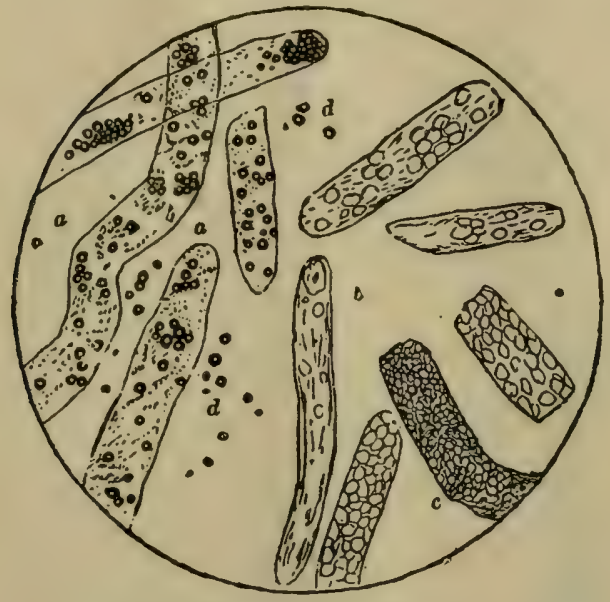

Fig. 26. a. Fatty casts. b. and c. Blood casts. d. Free fatty Molecules. (Roberts). have a hyaline matrix more or less concealed by epithelial cells. The presence of these casts is indicative of an acute process.

Blood casts consist of the hyaline matrix with blood corpuscles imbedded in or adhering to the matrix. Pus casts are rare, but when present the pus corpuscles adhere to the matrix.

Blood casts are indicative of a hemorrhage into the tubules and of an acute hemorrhagic process. Hyaline and epithelial casts are usually associated with them.

Granular casts usually have well defined boundaries with granular matter imbedded in or adhering to the matrix. They may be finely or coarsely granular, the latter having a more serious significance. Granular casts are due to a disintegration of the renal epithelium. Their

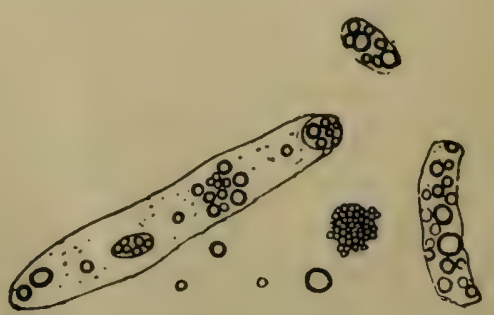

FIg. 27. Fatty Casts and Fat Droplets. 

degree of refraction is changeable; sometimes they appear yellowish, at other times colorless.

Fatty casts have a hyaline matrix containing a number of small, glistening fat globules and granules. Some free fat is also usually found in the field. As fatty casts are secondary products of epithelial and granular casts, the diagnosis of a chronic process is justifiable. The hyaline matrix is characteristic of the different varieties of casts that have been mentioned up to this point.

Waxy casts differ in chemical composition from those previously mentioned. They are characterized by wavy contours; a high refracting power; a more or less yellowish color and quite a high degree of brittleness. They are slowly, if at all, attacked

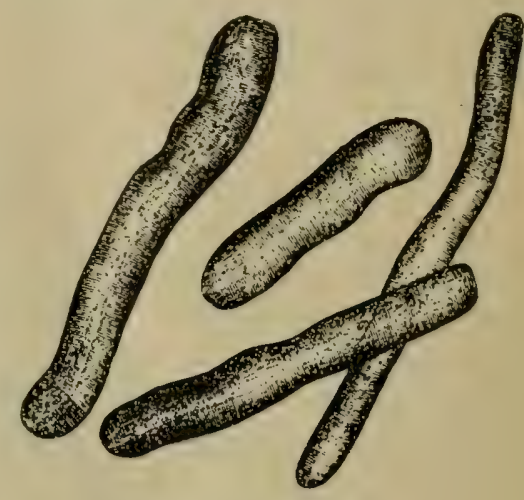

FIG. 28. Waxy Casts. by acetic acid. Their presence signifies waxy degeneration of the kidney. Hyaline casts may sometimes have a superficial resemblance to waxy casts, but they never have the same high refraction as the latter.

In the urine of the horse, the sediment of crystals of lime carbonate may obscure the search for casts. In this case add a few drops of acetic acid which will dissolve the crystals quickly, and the casts, if present, will show more distinctly but undergo solution more slowly than the crystals.

Cast-like formations are composed of various elements having a form somewhat similar to casts but lacking the matrix soluble in acetic acid. Amorphous urates often simulate granular casts in form. Bacteria are often grouped in a manner similar to the form of cast, but a close inspection shows an irregular outline, and usually a number of groupings not in cast form. Granular detritus and hematoidin may also assume the form of casts; likewise epithelial cells, blood corpuscles and fibrin in renal hemorrhages may also assume the form of casts. Acetic acid is said to be a reliable reagent for differentiating between true and false 

casts. This reagent dissolves the matrix of the true casts but does not act upon the cast-like formations.

Cylindroids appear like hyaline casts, but are large and band-like. Their breadth is uniform and they often contain

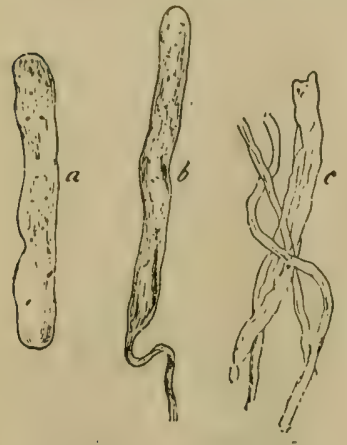

FIG. 29. Cylindroids. crystals, epithelial cells and corpuscles. They are soluble in acetic acid and are of renal origin. No special significance is attached to them.

Mucous cylinders sometimes called cylindroids are usually not of uniform breadth and seldom contain morphologic constituents and are insoluble in acetic acid. They are usually found in any urine containing an abundance of mucus and are of no special significance.

a. b, Cast-like forms: c.

Filamentous forms. (Ogden).

Upon special blanks, test specimens of urine quantitatively, the number and amount of constituents being unknown. 

Form used in examination of Horse Urine.

Owner Address

No . Species Age Sex

Object Date

\begin{tabular}{lll}
\hline & Normal (Horse). & Sample \\
Amount in 24 hours & $3000-4000$ c. c. \\
Specific Gravity & $1025-1050$ \\
Reaction & Alkaline \\
Color & Yellowish brown \\
Translucency & Turbid \\
Consistency & Viscid & \\
Total Solids & $50-120$ parts per 1000 \\
$\quad$ Chlorides & $8-14$ ". \\
$\quad$ Sulphates & $2-3$ " & " \\
$\quad$ Phosphates & $.05-.2$ " & \\
Urea & $20-40$ " & \\
Uric Acid & Trace & \\
Hippuric Acid & $4-8$ parts per 1000 \\
Indican & $.1-.2$ "
\end{tabular}

ABNORMAL CONSTITUENTS.

Albumin

Sugar

Bile

Hemoglobin

MICROSCOPIC EXAMINATION.

Epithelial Cells

Leucocytes

Blood

Casts

Spermatozoa

Micro-crganisms

Calcium Carbonate

Crystalline Deposit.

Calcium Oxalate

Triple Phosphates 

Form used in Examination of Human Urine.

Name

Address

Date.

Normal.

Sample.

Amount in 24 hours. $1000-1500 \mathrm{cc}$.

Specific gravity

$1015-1025$

Reaction

Acid $\left(30^{\circ}-40^{\circ}\right)$

Color

Translucency

Light golden

Sol ds

Clear

Chlorides

34-50 parts per 1000

50-75 parts per 24 hours

6-9 parts per 1000 as $\mathrm{NaCl}$

10-15 parts per 24 hours

$1.5-2.5$ parts per 1000 as $\mathrm{P}_{2} \mathrm{O}_{5}$

Phosphates

2. -3.5 parts per 24 hours

Sulphates

1.5-3 parts per 1000 as $\mathrm{SO}^{3}$

Urea

2.5-4 parts per 24 hours

14-22 parts per 1000

Uric Acid

22-33 parts per 24 hours

0.25-0.40 parts per 1000

$0.40-0.60$ parts per 24 hours

Indican

Normal, little, medium, much

Albumin

Sugar

Bile

Hemoglobin

MICROSCOPIC EXAMINATION.

Epithelial cells

Leucocytes

Blood

Casts

Uric Acid

Crystalline Deposit.

Oxalates

Triple Phosphates

Urates 



\section{Procedure in Examining a Sample of Urine.}

Experience has shown that the following procedure expedites the work of examination. Pour the urine into a sedimentation glass, as some little time may be required for the sediment to settle. After noting the amount, color and translucency, take the specific gravity with the urinometer. This may be done in the sedimentation glass. Filter as much of the urine as may be needed for the subsequent tests. While waiting for the urine to filter make the tests for urea and uric acid as the correct reading is not obtained until some few minutes after the tests have been made. Test the reaction with litmus paper or if the degree of acidity is required (human) use the acidimeter. Test for indican. Thus far the tests may be made just as well with the unfiltered as the filtered urine.

For the albumin and sugar tests the filtered urine must be used, using two or three different tests for each substance. If albumen is present it should be removed before making the sugar tests. Also before making the centrifuge tests for the chlorides, phosphates and sulphates. This may be done by adding a little acetic acid to the urine and boiling it until the albumin is coagulated. Filter out the albumin and use the urine thus filtered for the remaining tests.

With a pipette remove some of the sediment at the bottom of the sedimentation glass and introduce it into a centrifuge tube filling the remainder of the tube to the required height with the unfiltered urine. Include this with the other centrifuge tubes used in making the tests for the chlorides, etc., revolving them in the centrifuge for three minutes. If albumin is present and it is desired to know the amount, it may be determined by the centrifugal method as described in the text.

For the microscopial examination, prepare a couple of slides from the sediment in the centrifuge tube, after pouring off the clear urine above. It is well, also, to prepare a slide or two from the sediment in the sedimentation glass. After putting a drop or two of the sediment on the slide it may be covered witha cover glass and examined clear or before covering the sediment a drop of dilute Lugol's solution, or safranin or other stain may be added. In some cases the slight tint of the dye appears to make the casts or other elements stand out more clearly. To detect the amyloid bodies or glycogen cells it is necessary 

to use the iodine solution and this often times serves to make the casts and other elements more distinctly visible.

\section{APPENDIX.}

Formulae for Reagents.

Barium Chloride Solution for Centrifuge Sulphate Test.

$\begin{array}{ll}\text { Barium chloride, } & 4 \text { parts } \\ \text { Hydrochloric acid, } & 1 \text { part } \\ \text { Distilled water, } & 16 \text { parts }\end{array}$

Barium Chloride Solution for Quantitative Test of Sulphates.

Pure crystallized barium chloride,

Distilled water to

30.5 grams. 1000. cc.

Baryta Mixture. This mixture is prepared by making saturated solutions in the cold, of barium nitrate and barium hydrate, and adding two volumes of the hydrate to one volume of the nitrate.

\section{Benedict's Solution.}

Solution 1. Cupric Sulphate

Distilled water up to

34.65 grams

500 . cc.

Solution 2. Sodium carbonate (anhydrous),

Rochelle salt,

100. grams

Distilled water up to

173. grams

500. cc.

Benedict's Single Solution.

Cupric Sulphate,

17.3 grams

Sodium Citrate,

173. grams

Sodium Carbonate (anhydrous),

100. grams

Distilled water up to

1000. cc.

Benedict's Solution for Quantitative Tests.

Copper Sulphate Crystals,

18 grams

Sodium Carbonate Crystals,

200 grams

Sodium Citrate,

200 grams

Potassium Thiocyanate,

125 grams

Potassium Ferrocyanide (5\% \%ol.) $5 \mathrm{cc}$.

Distilled water to $1000 \mathrm{cc}$.

Dissolve the citrate, carbonate and thiocyanate in $800 \mathrm{cc}$. 

of distilled water with the aid of heat. Dissolve the copper sulphate separately in $100 \mathrm{cc}$. of distilled water and mix the two solutions slowly with constant stirring; then add the ferrocyanide solution. Cool and dilute with distilled water to exactly $1000 \mathrm{cc}$.

Cochineal Solution. Boil 40 grams of cochineal in $800 \mathrm{cc}$. of water. When cool add $200 \mathrm{cc}$. of alcohol and filter.

Decinormal Oxalic Solution. Dissolve 6.285 grams of pure oxalic acid in enough distilled water to make at or near $15^{\circ} \mathrm{C}$. $\left(59^{\circ} \mathrm{F}\right.$.), exactly $1000 \mathrm{cc}$.

Decinormal Sodium Hydroxide Solution. Dissolve 3.996 grams of pure sodium hydroxide in enough distilled water to make, at or near $15^{\circ} \mathrm{C}$. $\left(59^{\circ} \mathrm{F}\right.$.), exactly $1000 \mathrm{cc}$.

Ehrlich's Diazo-reaction.

Solution 1. Sulphanilic acid,

Hydrochloric acid,

Distilled water,

2 grams

$50 \mathrm{cc}$.

$1000 \mathrm{cc}$.

Solution 2. A $0.5 \%$ solution of sodium nitrite. Use in the proportion of 1 part of No. 2 to 50 parts of No. 1 .

Esbach's Reagent.

Picric acid,

Citric acid,

Distilled water up to

10 grams

20 grams

$1000 \mathrm{cc}$

\section{Fehling's Solution.}

Solution A. Pure Copper Sulphate crystalline, 34.64 grams Distilled water up to 500. cc.

Solution B. Sodio-potassium tartrate (Rochelle salt),

Pure caustic potash,

Distilled water up to

173. grams

125. grams

500. cc.

Use equal parts of $\mathrm{A}$ and $\mathrm{B}$.

Hypobromite Solution. Dissolve 40 grams of caustic soda in $100 \mathrm{cc}$. of distilled water. To $23 \mathrm{cc}$. of this solution add $2 \mathrm{cc}$. of bromine. To this mixture add an equal volume $(25 \mathrm{cc}$.) of water. The solution is not very stable and is best made up as needed. 

Lugol's Solution.

Iodine,

Potassium iodide,

Distilled water to
2.5 grams

5. grams

50. cc.

This may ordinarily be diluted 1 to 5 or 10 for urine work.

Magnesia Mixture.

Magnesium sulphate,

1 part.

Ammonium chloride,

1 part

Ammonia water,

1 part

Distilled water,

8 parts

\section{Millard-Roberts Reagent.}

Nitric acid,

1 part

Sat. Sol. Magnesium Sulphate, 5 parts

Mohr's Volumetric Method for Chlorides.

Solution 1. Fused silver nitrate,

29.075 grams

Distilled water to make, 1000 . cc.

Solution 2. Neutral potassium chromate, 10. grams

Distilled water to make, $100 . \quad$ cc.

Nylander's Reagent. Digest 2 grams of bismuth subnitrate and 4 grams of Rochelle salt in $100 \mathrm{cc}$. of a $10 \%$ solution of potassium hydroxide. The reagent should then be cooled and filtered.

Obermayer's Reagent. Dissolve 2 grams of solid ferric chloride in 500 cc. of concentrated hydrochloric acid. Sp. gr. 1.19.

Phenolphthalein. Dissolve 1 gram of phenolphthalein in $100 \mathrm{cc}$. of $95 \%$ alcohol.

\section{Ruhemann's Uric Acid Reagent.}

Iodine,

Potassium iodide,

Absolute alcohol,

Glycerine,

Distilled water to
0.5 grams

1.25 grams

7.5 grams

5. grams

100. grams

Salicylic Adehyde.

Dissolve 1 gram of salicylous acid in $10 \mathrm{cc}$. of absolute alcohol.

Shieb's Reagent.

Solution 1. Ammonium Sulphate (purest), 1.2 grams

Copper Sulphate (purest), 2.6 grams

Distilled water up to

50. cc. 

Solution 2. Caustic potash C. P. 20. grams

Distilled water up to

50. cc.

Dissolve and when cool, add

Glycerine,

50. cc.

Ammonia water, 0.960 sp. gr. 300. cc.

Add No. 1 to No. 2 and dilute the whole. to $500 \mathrm{cc}$. with distilled water. Stopper securely and shake until thoroughly mixed. 









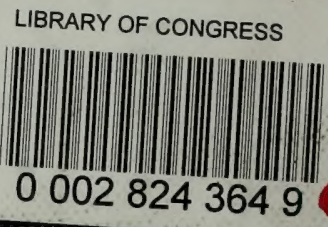

